\title{
An Ultra Wideband Survey: Global Regulations and Impulse Radio Research Based on Standards
}

\author{
Ville Niemelä, Student Member, IEEE, Jussi Haapola, Matti Hämäläinen, Senior Member, IEEE and \\ Jari Iinatti, Senior Member, IEEE
}

\begin{abstract}
This article presents an updated survey on research related to Ultra Wideband (UWB) communications, particularly that of impulse radio (IR) technology. In addition to the research, we survey UWB physical layer (PHY) specifications of the two existing standards - the IEEE 802.15.6-2012 and the IEEE 802.15.4-2015 - as well as the leading global UWB spectrum regulatory limitations which have been updated recently. The latter standard including the UWB specifications was first published in 2007 and the latest revision dates to 2015. The focus in this article is the period from 2007 to 2015 . Our purpose is to provide an in-depth survey with a clearly specified topic together with the standard specifications and the related regulatory restrictions. Additionally, the last part of the article discusses the possibilities of increasing the current IR-UWB data rates to meet increasing future demands.
\end{abstract}

Index Terms-IEEE 802.15.6-2012, IEEE 802.15.4-2015, IRUWB, Physical Layer, UWB Regulations, WBAN, WPAN

\section{INTRODUCTION}

$\mathrm{T}$ HE abbreviation UWB (ultra wideband) was apparently first defined by the Office of the Secretary of the Defense/Defense Advanced Research Project Agency (OSD/DARPA) in the late 1980s [1]. The research on the topic, however, had been ongoing for decades by then [2]. During the 1990s, there was an increasing interest for the UWB research with a few milestone studies [3] [4] and in the beginning of the 2000s, the Federal Communications Commission (FCC) became the first authority to define regulations for the use of UWB technology [5]. Europe [6], Japan [7], and a few other Asian countries followed a couple of years later [8].

An attempt to standardize UWB was made in 2003 by the IEEE 802.15.3a task group, but it was withdrawn as no unanimous decision was reached between two competing proposals which were the multiband (MB) and the impulse radio (IR) approaches [9] [10]. The multiband approach was later included into another standard called ECMA-368 [11]. In

Manuscript received March 11, 2016; revised August 12, 2016; accepted November 25, 2016. This work was supported in part by the Finnish Funding Agency for Innovation (Tekes) within the project EWiHS and the Academy of Finland within the project DWHN.

All the authors are with the Centre for Wireless Communications (CWC), University of Oulu, Oulu, Finland. (email: firstname.lastname@ oulu.fi).
2007, the IEEE published its first standard that included UWB specifications, the IEEE 802.15.4a-2007 which was an amendment to the IEEE 802.15.4-2006 targeted for low datarate wireless personal area networks (WPANs). The two WPAN standards were first updated and merged together in 2011 and it is currently known as the IEEE 802.15.4-2015 [12]. The IEEE published another UWB standard in 2012, known as the IEEE 802.15.6-2012, which is targeted for wireless body area networks (WBANs) [13]. At the moment, these two standards are the only ones including IR-UWB physical layer (PHY) specifications, among other PHYs. The IEEE 802.15.6-2012, i.e. the WBAN standard, includes also a frequency modulated (FM) option for the UWB.

In the past decade numerous articles with different approaches of research on UWB and on the standards mentioned above have been published. Among the first ones were [14] and [15], which considered different features of the first release of the WPAN standard from 2007. Both compare briefly the frequency band allocation specified by the standard to the existing regulatory definitions at that time. More indepth studies, e.g., [16] and [17] appeared a couple of years later with the fashion acronym of the 2000s: wireless sensor network (WSN). Besides the UWB PHY aspect, both of these papers covered medium access control (MAC) specifications of the IEEE 802.15.4a. Additionally, in [16], the global regulations including US, Europe and Japan versions are presented in detail. The paper [17] also included the chirp spread spectrum PHY specifications of the standard.

Similarly, related to the WBAN standard, the first publications presenting its essential features were overview type of papers with brief descriptions of the standard, e.g., [18] and [19], followed by more in-depth studies later on [20], [21]. All of the numerous articles [14] - [21] dealt with one standard only and with multiple topics, for example, PHY and MAC or several different PHYs. The article [16] from 2009 is the only one presenting a broad literature review. None of the articles [14] - [21] presented a compilation where the two existing standards' specifications regarding UWB PHY and the most important regional regulatory definitions concerning UWB can be found jointly together with a comprehensive survey. This type of compilation is the main contribution of this survey. Considering that there are recent updates in the standard specifications and in the regulatory limitations, it is 
appropriate to update the literature review as well.

Concerning short-range communications that WPAN and WBAN standards are aimed for, particularly the WBAN has been very popular topic over the past few years. Many WBAN related surveys have in the recent years considered UWB as one option for short range technology, e.g., [22] - [25]. However, they focus mainly on other topics than the UWB or present only one of the existing UWB standard solutions and do not address the UWB regulatory definitions which have been updated in the recent years.

There are also articles focusing on specific topics [21] and [26] - [28] providing truly in-depth presentations. This article aims to do the same by providing a joint review presentation of the named two IEEE standards while focusing on UWB PHY to provide in-depth information of the topic. The main importance of the joint presentation is for future Internet-ofThings (IoT) network compatibility and usability. By specifying the differences and the similarities of the two UWB PHYs it is easier to design and to construct a WPAN-WBAN dual-radio chip. These chips could improve optimization of the data flow from a sensor to a core network and database and could provide accurate ranging at the same time. A joint review of the two standards with comparisons helps also in understanding the weaknesses and the strengths of the both standards. The focus is on the period after the first release of the IEEE 802.15.4a in 2007. The main new contribution of this article is the discussion and the recognition of the potential increase of IR-UWB data rate within the two existing standards.

Since our target is to provide a clearly specified survey related to IR-UWB communications instead of an extremely wide coverage of many possible topics, certain approaches are excluded. First, UWB channel and propagation issues which have been quite thoroughly covered in the recent years, e.g., in [27] and [29], thus their reference lists offer a number of indepth articles to the interested reader. Second, MAC related studies are not discussed as it is an extensively wide topic area on its own having resulted in several recent detailed articles, e.g., [30] - [32]. To summarize, the main contributions of this article are:

- Presentation of the recently updated global regulations for indoor UWB radiation limits,

- Description of both UWB PHYs of the existing IEEE standards in an in-depth manner,

- Comparison of the UWB PHYs of the two standards,

- Presentation of a comprehensive literature survey on IRUWB research from the past ten years,

- Discussion of the future data rate potential of the IRUWB technology.

The remainder of this article is organized as follows. The next section presents the current UWB options and briefly compares their data rates and frequency bands to the two most popular short range technologies. Section III presents the updated UWB regulations of US, Europe and Japan together with the corresponding UWB frequency band plans of the two IEEE standards. In Sections IV and V, the UWB PHYs of the
IEEE 802.15.6-2012 and IEEE 802.15.4-2015 are described, respectively. In Section VI, a short comparison of the standards is presented. Section VII provides a literature review of the research related to UWB and the two standards. Section VIII presents some considerations of the future data rate capability and scalability of the IR-UWB concerning the defined modulations and the symbol structure. Finally, Section IX concludes the article with a table listing all the used acronyms.

\section{DifFERENT TeChNOLOGIES FOR SHORT-RANGE COMMUNICATIONS}

There are several different wireless technologies that can be used for short-range technologies. Here, we briefly compare two popular and currently widely used protocols, Bluetooth (versions 1.1-4.0), and ZigBee, standardized as the IEEE 802.15.1 and IEEE 802.15.4, respectively, to the UWB options that currently exist. In addition to the UWB options defined by the two standards dealt with in this article, multiband orthogonal frequency division modulations (MBOFDM) UWB also exists providing data rates up to $480 \mathrm{Mbps}$ [11]. The selected short-range technologies are presented in TABLE I with their data rate capabilities and approximate frequency utilization.

TABLE I. Comparison of different short-range communication technologies

\begin{tabular}{|l|l|l|}
\hline Technology/standard & Data rate & Frequency band \\
\hline \hline $\begin{array}{l}\text { IR-UWB/ } \\
\text { IEEE 802.15.4-2015 }\end{array}$ & $0.11-27 \mathrm{Mbps}$ & $\begin{array}{l}<10.6 \mathrm{GHz} \text { with regulatory } \\
\text { restrictions }\end{array}$ \\
\hline $\begin{array}{l}\text { IR-UWB/ } \\
\text { IEEE 802.15.6-2012 }\end{array}$ & $0.49-15 \mathrm{Mbps}$ & $\begin{array}{l}<10.6 \mathrm{GHz} \text { with regulatory } \\
\text { restrictions }\end{array}$ \\
\hline $\begin{array}{l}\text { FM-UWB/ } \\
\text { IEEE 802.15.6-2012 }\end{array}$ & $0.25 \mathrm{Mbps}$ & $\begin{array}{l}<10.6 \mathrm{GHz} \text { with regulatory } \\
\text { restrictions }\end{array}$ \\
\hline $\begin{array}{l}\text { MB-OFDM-UWB/ } \\
\text { ECMA-368 }\end{array}$ & up to 480 Mbps & $\begin{array}{l}<10.6 \mathrm{GHz} \text { with regulatory } \\
\text { restrictions }\end{array}$ \\
\hline ZigBee (IEEE 802.15.4) & $\begin{array}{l}0.02,0.04 \text { and } \\
0.25 \mathrm{Mbps}\end{array}$ & $\begin{array}{l}868 \text { and } 915 \mathrm{MHz}, 2.4 \mathrm{GHz} \\
\text { ISM }\end{array}$ \\
\hline $\begin{array}{l}\text { Bluetooth (IEEE } \\
\text { 802.15.1) }\end{array}$ & $1-24 \mathrm{Mbps}$ & $2.4 \mathrm{GHz} \mathrm{ISM} \mathrm{and} \mathrm{5} \mathrm{GHz}$ \\
\hline
\end{tabular}

There are numerous articles in current literature that include a comparative approach on short-range wireless communications. For example, both [23] and [33] provide extensive surveys of the currently available technologies with the main focus on WBAN requirements and challenges. They include different versions of Bluetooth, ZigBee, MB-OFDMUWB among many other options. Implemented systems, on the other hand, are studied and compared in [26] from the medical and healthcare perspectives including both Bluetooth and ZigBee based systems, among a few others.

One of the latest articles comparing Bluetooth, ZigBee, and IEEE 802.15.4 based IR-UWB is [34], where the main approach is energy consumption together with transmission time and data coding efficiency. Based on the simulation results among the selected features, as introduced in this article, IR-UWB seems to perform better than Bluetooth and ZigBee. Security issues are studied in [35] examining Bluetooth, ZigBee, MB-OFDM-UWB and Near Field Communication (NFC) technologies. Each of these 
technologies have challenges, e.g., against eavesdropping and improving security by encryption and authentication which requires additional hardware thus increasing the complexity and power consumption of the system. However, it is predicted in [35] that the studied UWB technology could provide a power and a cost-effective solution for these challenges.

\section{UWB REGULATIONS AND THE STANDARDS' FREQUENCY BAND PLANS}

Global regulations regarding UWB spectrum allocation are diverse. The only conjunctive factor is the maximum mean equivalent isotropic radiated power (EIRP) level of the UWB transmitter, namely $-41.3 \mathrm{dBm} / \mathrm{MHz}$. The spectrum available for UWB devices for transmission on this level varies quite a lot.

The UWB EIRP mask for indoor devices regarding US [5], Europe [6], and Japanese [7] regulatory bodies is presented in Fig. 1 with two subplots. In the upper subplot, the US and the Japanese UWB masks are presented and in the lower one, the US and the European masks. The frequency band allocation for the UWB of the two IEEE standards is presented in Fig. 2. The upper subplot presents the IEEE 802.15.4-2015 [12] band allocation and the lower subplot the IEEE 802.15.6-2012 [13] allocation.

The US mask from 2002 by the FCC [5] is the most liberal one from the allowed spectrum width point of view. The maximum mean EIRP of $-41.3 \mathrm{dBm} / \mathrm{MHz}$ can be used from 3.1 $\mathrm{GHz}$ up to $10.6 \mathrm{GHz}$. Moreover, the UWB transmissions with the same EIRP level are also allowed below $960 \mathrm{MHz}$. The FCC defines a signal as UWB if the minimum bandwidth is $500 \mathrm{MHz}$ or if the fractional bandwidth is at least 0.2 [5].

The Japanese UWB mask [7], originally from 2006 and updated in 2008, 2010, and 2015, has the same maximum mean EIRP as the US UWB mask, but the available spectrum width is much narrower. There are two different bands allowed for the maximum mean EIRP transmissions, the low band of $3.4-4.8 \mathrm{GHz}$ and the high band of $7.25-10.25 \mathrm{GHz}$. The low band is, according to the latest update, available only for devices utilizing interference mitigation while the high band can be used without mitigation techniques. Note that, according to the Japanese definition the minimum required bandwidth for a UWB signal is $450 \mathrm{MHz}$.

The European UWB mask [6] from 2006 with two updates from 2007 and 2011 has also two possible bands with the same maximum mean EIRP limit. The bands are between 3.1 $-4.8 \mathrm{GHz}$ and $6-9 \mathrm{GHz}$, but there are a few limitations. The low band of $3.1-4.8 \mathrm{GHz}$ can be used in Europe only if a UWB device is utilizing either detect and avoid (DAA) technology or low duty cycle (LDC). Another exception is the band of $8.5-9 \mathrm{GHz}$ which can be used only if DAA is used. Therefore, the band of $6-8.5 \mathrm{GHz}$ is the only option for UWB transmissions in Europe if no mitigation techniques are utilized.
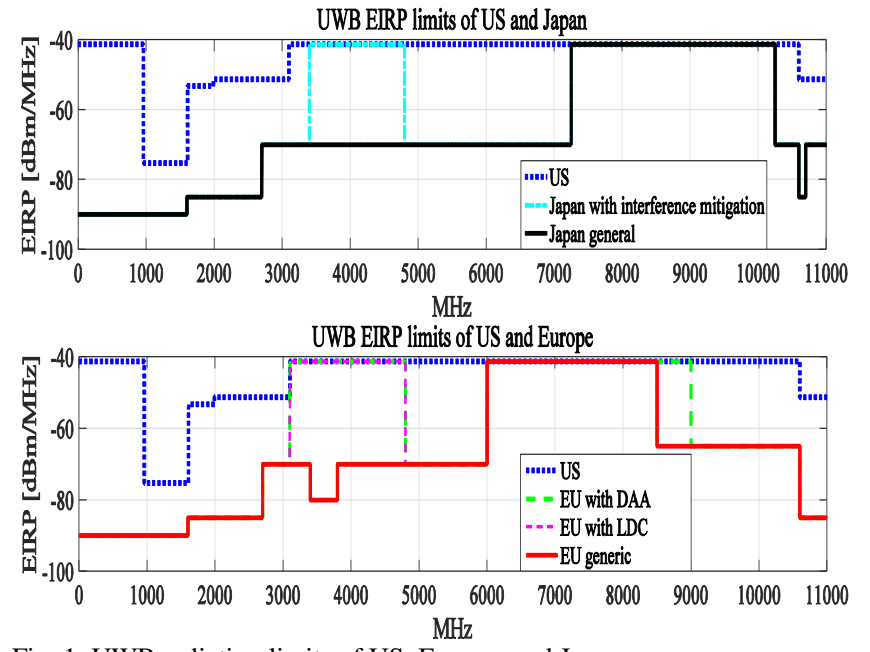

Fig. 1. UWB radiation limits of US, Europe, and Japan.

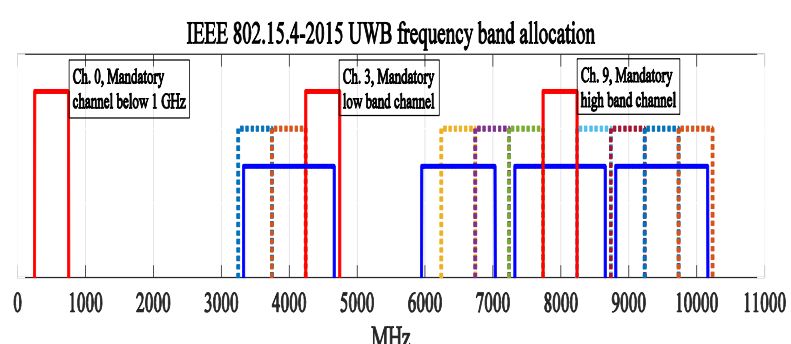

IEEE 802.15.6-2012 UWB frequency band allocation

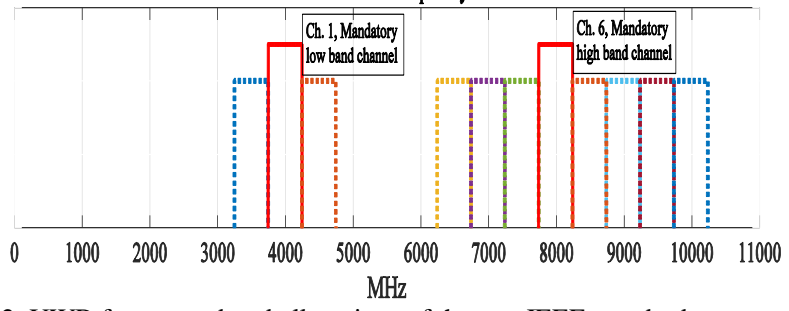

Fig. 2. UWB frequency band allocations of the two IEEE standards.

The only common frequency band that can be used for indoor UWB transmission within the limitations set by all the three regulatory bodies, when no mitigation techniques are used, is between $7.25-8.5 \mathrm{GHz}$. If the DAA or LDC is applied, the possible band is extended to $3.4-4.8 \mathrm{GHz}$ and to $8.5-9 \mathrm{GHz}$.

Regarding the IEEE 802.15.4-2015, the frequency band allocation is divided to sub-gigahertz band and to low and high bands. The corresponding frequency bands are $250-749$ $\mathrm{MHz}, 3.25-4.74 \mathrm{GHz}$ and $5.95-10.23 \mathrm{GHz}$, respectively. The total number of channels is 16 with three mandatory channels, one in each band that needs to be supported in order to be compliant with the standard.

When compared to the IEEE 802.15.6-2012, the most significant difference is the absence of the sub-gigahertz band. Another difference is that the IEEE 802.15.6-2012 limits the bandwidth of a channel to $499 \mathrm{MHz}$ as for the IEEE 802.15.42015 , it is possible to use channels with over one gigahertz of bandwidths, e.g., channel \#15 with $1.35 \mathrm{GHz}$ of bandwidth. The IEEE 802.15.6-2012 is also divided to low and high bands with corresponding frequency bands of $3.25-4.74 \mathrm{GHz}$ and 
$6.24 \mathrm{GHz}-10.23 \mathrm{GHz}$, respectively. There are 11 channels with two mandatory channels, one in each band that needs to be supported in order to be compliant with the standard. The highest bars present the mandatory channels in Fig. 2.

\section{IEEE 802.15.6-2012 UWB PHYSICAL LAYER}

Presented in the standard [13] and in many articles [20], [21], [23], [24], the IEEE 802.15.6-2012 includes three different PHYs: human body communication (HBC), UWB and narrowband. The UWB PHY is further divided into two different categories, IR-UWB and FM-UWB. Moreover, there are two different modes of operations in the UWB PHY which cross-use the IR- and FM-UWBs. These are the default mode and the high-quality of service mode (high QoS). As pointed out by the authors of [21], the UWB PHY can also be considered from two interlinked aspects: the construction of the UWB frame format and the transformation of formatted bits to radio signals. This article follows the same approach.

\section{A. The Use of UWB in a BAN}

The IEEE 802.15.6-2012 defines a BAN to construct of a hub and devices. The use of IR-UWB and FM-UWB in a BAN is further defined as follows [13]:

- A hub shall implement IR-UWB transceiver only or

- A hub shall implement both IR-UWB and FM-UWB transceivers.

For a device, the definitions are a bit different:

- A device shall implement IR-UWB transceiver or

- A device shall implement FM-UWB transceiver or

- A device shall implement both IR-UWB and FM-UWB transceivers.

The definitions lead to a conclusion that in an IEEE 802.15.6-2012 [13] BAN, a hub always needs to support IRUWB whether it is the used technology or not, as the devices can be built to use only one of the two UWB options or both.

\section{B. Modes of Operation}

The standard's [13] default mode of operation is generally intended for a wide range of applications which can be medical or non-medical. The high QoS mode of the standard is intended for medical applications which require high priority class-based communications.

Regarding the modes, there are several mandatory procedures defined for interoperability. In both modes, the mandatory supported UWB PHY is IR. In the default mode, the FM-UWB is defined as an optional PHY but in the high QoS mode, an optional PHY is not precluded. Hence, it can be interpreted that other UWB PHY options with different modulations can be used in the high QoS as long as the mandatory PHY is supported, too.

For the FM-UWB, there is only one option for modulation (combination of continuous phase binary frequency-shift keying (CP-BFSK) and wideband FM) and for the data rate (250 kbps, uncoded) and thus, they are set mandatory.

For the IR-UWB, the modulation options include:

- On-off signaling, (on-off keying, OOK) mandatory modulation in the default mode. Note that on-off signaling with the half-rate symbol mapper combined with 2-ary waveform coding defined in the standard is equal to pulse position modulation (PPM).

- Differential encoding with binary or quadrature phaseshift keying (DBPSK/DQPSK), mandatory modulation in the high QoS.

Other mandatory procedures listed, which are the same for both modes, include the physical layer protocol data unit (PPDU) and the transmitted spectral mask, which must follow the same specifications. On the other hand, the mandatory required channels are partially the same. When utilizing FMUWB in the default mode, there is only one mandatory channel in the high band (Ch. \#6). With IR-UWB in the both modes, there is one mandatory channel in the low band $(\mathrm{Ch}$. \#1) and one in the high band (Ch. \#6). For fulfilling the requirements, using one mandatory channel only is sufficient.

\section{UWB Frame Format}

The UWB PHY frame format (the PPDU) is constructed by concatenating synchronization header (SHR), physical layer header (PHR), and physical layer service data unit (PSDU). Also, the transmission of the bits in the PPDU is performed in this order. Fig. 3 presents a schematic diagram of the UWB PHY and it is divided into two parts: UWB frame formatting and bit transformation into radio signal. The bits formed in the MAC layer are called the MAC protocol data unit (MPDU) consisting of the MAC header, the MAC frame body containing the actual data bits, and the frame check sequence. The MPDU is further configured to form the PSDU. The first step is the additive scrambler mixing the bit stream to eliminate possible long strings of $0 \mathrm{~s}$ and $1 \mathrm{~s}$. The receiver uses the same initialization vector as the transmitter to de-scramble the bit stream. The information of the vector is carried by the PHR.

The Bose-Chaudhuri-Hocquenghem (BCH) code with $n=$ 63 and $k=51$ is used in the default mode for forward error correction (FEC), where $k$ is the number of data bits and $n$ is the length of the code word. In the high QoS mode, the used code is $\mathrm{BCH}(n=126, k=63)$ together with hybrid type II automatic repeat request. The correction ability of the $\mathrm{BCH}$ $(63,51)$ is 2 data bits and of the $\mathrm{BCH}(126,63) 10$ data bits.

After the BCH-FEC, pad bits are utilized for aligning a symbol boundary and the appended pad bits are set to zero.

The last step in PSDU construction is bit interleaving providing robustness against error propagation. The correction capability of the $\mathrm{BCH}$ code is limited, especially against burst errors. Adopting the bit interleaving, the burst errors can be handled in a more effective manner.

After the PSDU is constructed, the PHR is added to it and the transformation into the radio signal follows. The PHR frame is formed of 24 bits containing information on the used data rate, the pulse type and the length of the MAC frame body, to name a few. Header check sequence is added to the PHR frame and then the shortened BCH $(n=40, k=28)$ code is applied to form the PHR itself in the default mode. In the high QoS mode, the shortened BCH $(n=91, k=28)$ code is applied. Both the PHR and the PSDU are handled in the same 
manner concerning the transmission rate and the pulse shaping. These, however, depend on the modulation specific rulings and the chosen timing parameters.

UWB frame formatting

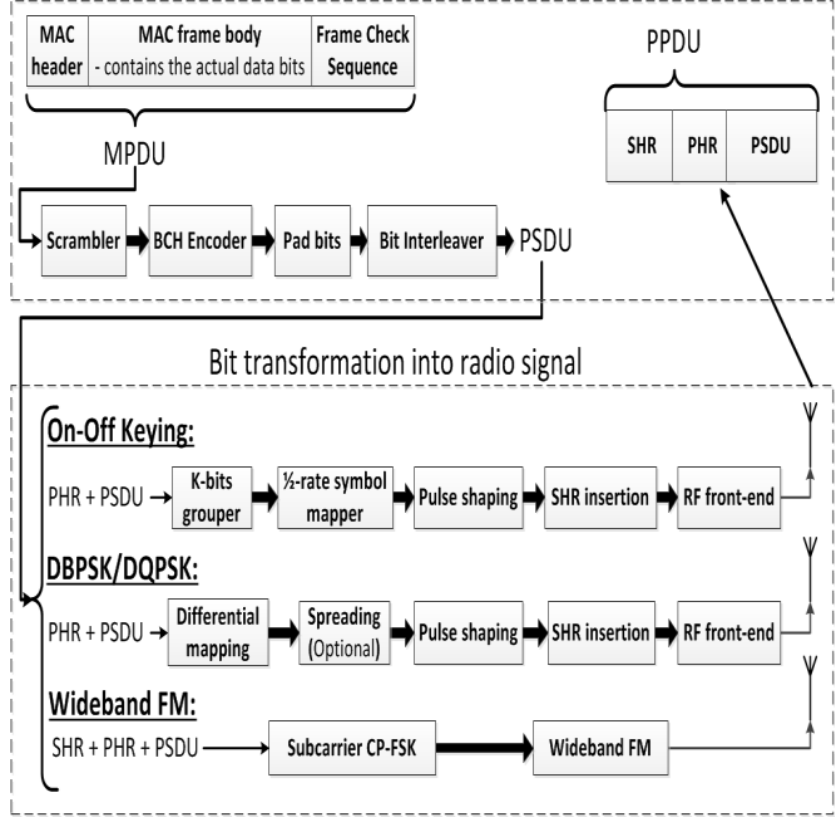

Fig. 3. Schematic diagram of the UWB PHY of the IEEE 802.15.6-2012.

The SHR is handled differently when compared to the transmission of the PHR and the PSDU. In OOK and DBPSK/DQPSK, the SHR is inserted right before the transmission as in FM-UWB it is inserted together with the PHR.

In OOK and DBPSK/DQPSK, the SHR consists of a preamble and a start-of-frame delimiter (SFD). The SHR is formed based on a 5-time repetition of symbol $S_{i}$. In the preamble part, the symbol $S_{i}$ is transmitted four times and in the SFD once. The symbol $S_{i}$ is constructed of a 63 bit Kasami sequence with zero-padding each bit by $L-1$ zeros. In OOK, $L=16$ and in DBPSK/DQPSK, $L=32$. In the SFD, the $\mathrm{S}_{i}$ is a bit-wise inversion of the symbol sent in the preamble part to provide low cross-correlation within the two SHR parts. The SHR is always transmitted with the same rate, $3.90 \mathrm{Mbps}$, regardless of the chosen rate for the PHR and the PSDU. The SHR rate is due to the set pulse/burst duration, $8 \mathrm{~ns}$, and the number of zero-paddings, $L-1$, both specified in the standard [13].

In the FM-UWB, the SHR consists of the preamble only and there is no SFD. The same symbol $S_{i}$ is used but without zero-padding and it is sent only once. The used bit rate is the same and the only one defined for the FM-UWB, i.e. 250 kbps.

\section{Transformation of Formatted Bits into Radio Signals}

In the FM-UWB modulation (combination of CP-BFSK and wideband FM), the modulating-carrier signal can be either triangular, sine, or a sawtooth waveform. The information bearing signal contains a Gaussian pulse shape of bandwidthsymbol duration product of 0.8 .

In the IR-UWB, the waveform selection is divided to a single pulse option and a burst pulse option with three different pulse types. The single pulse option refers to one long pulse whereas the burst pulse option indicates to the traditional concept of IR-UWB with pulse duration typically less than 2 ns. The pulse types with different modulations and pulse options are as follows:

- Short pulse type:

$\circ$ OOK and DBPSK modulated burst of pulses. OOK can be used with one short pulse but it is still considered as burst pulse regardless that it coincides with the single pulse option. The DBPSK requires two pulses at the minimum for a waveform.

- Chirp pulse type:

- OOK and DBPSK/DQPSK modulated.

$\circ$ A baseband single pulse signal which frequency is swept up by $520 \mathrm{MHz}$ around the used center frequency during each transmission interval of the waveform.

- Chaotic pulse type:

- OOK modulated and single pulse option.

- Consists of pulses that are near constant envelope signals produced by the addition of different triangular or sawtooth waveforms.

Regarding the different pulse types, there is no mandatory pulse shape. However, the transmitted waveform needs to comply with the timing parameters defined in the standard and must fulfill the spectral mask requirements of the standard and the regulatory spectral mask where applicable (Fig. 1). The timing parameters are presented in TABLE II for OOK and in TABLE III for DBPSK/DQPSK.

Visible in TABLE III, $N_{\mathrm{cpb}}$ varies from 2 to 32 when the burst pulse option with DBPSK is applied. This does not apply to the single pulse option when $N_{\mathrm{cpb}}=1$ and it is possible to use DQPSK. Even though both DBPSK and DQPSK are considered and defined jointly in the standard, the DQPSK is intended for the chirp pulse type with the single pulse option.

\begin{tabular}{|c|c|c|c|c|c|c|}
\hline $\begin{array}{c}\text { Pulse repetition frequency (MHz) } \\
\text { and } \\
\text { uncoded bit rate (Mbps) }\end{array}$ & $\begin{array}{c}\text { Symbol duration } \\
T_{s y m}(\mathbf{n s}) \\
\end{array}$ & $\begin{array}{c}\text { Burst duration } \\
T_{w}(\mathrm{~ns})\end{array}$ & $\begin{array}{c}\text { \#Chips per burst } \\
N_{c p b}\end{array}$ & $\begin{array}{l}\text { FEC } \\
\text { rate }\end{array}$ & $\begin{array}{c}\text { \# Burst positions per symbol } \\
N_{w}\end{array}$ & $\begin{array}{c}\text { \# Hopping positions } \\
\text { per symbol } \\
N_{h o p} \\
\end{array}$ \\
\hline 0.487 (mandatory data rate) & 2051.300 & 64.103 & 32 & 0.81 & 32 & 16 \\
\hline 1.950 & 512.820 & 16.026 & 8 & 0.81 & 32 & 16 \\
\hline 3.90 & 256.410 & 8.012 & 4 & 0.81 & 32 & 16 \\
\hline 7.80 & 128.210 & 4.006 & 2 & 0.81 & 32 & 16 \\
\hline
\end{tabular}


TABLE III. IEEE 802.15.6-2012 timing parameters for DBPSK/DQPSK modulations

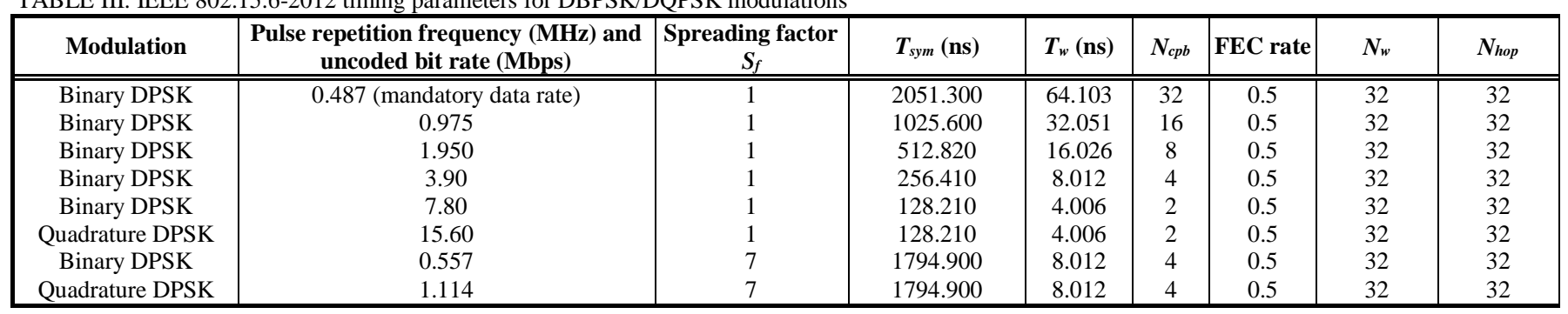

The IR-UWB symbol structure of the IEEE 802.15.6-2012 [13] is presented in Fig. 4. The symbol consists of $N_{\mathrm{w}}$ waveform positions and for the OOK it is divided into two halves. The duty cycle is limited to $3.125 \%$. Thus, there are $N_{\mathrm{w}}-1$ vacant positions in DBPSK and $N_{\mathrm{w}} / 2-1$ vacant positions in OOK. The vacant burst positions are used for multi-BAN coexistence support as there are $N_{\text {hop }}=N_{\mathrm{w}}$ hopping positions in DPSK and $N_{\text {hop }}=N_{\mathrm{w}} / 2$ hopping positions in OOK during one symbol interval.

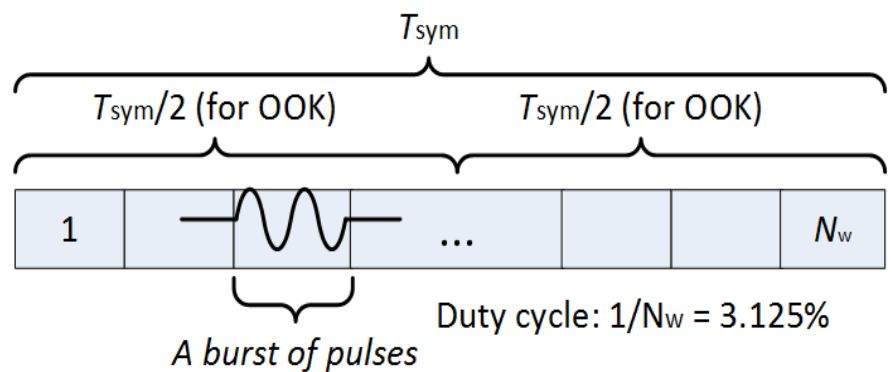

Fig. 4. UWB symbol structure of the IEEE 802.15.6-2012.

\section{1) On-Off Keying}

As presented in Fig. 3, after the PHR and the PSDU have been constructed in OOK, they are first grouped into $K$ bits. Following the $K$-bits grouper, a half-rate symbol mapper is utilized meaning that each binary data symbol is expressed as a binary code word of length $2 K$. When $K=1$, the defined binary data code words correspond to binary PPM providing some additional options at the detection on the receiver side.

In the burst pulse option, the transmitted waveform for OOK modulated signal in the $m^{\text {th }}$ symbol is [13]

$$
\begin{aligned}
x^{m}(t)= & \sum_{n=0}^{2 K-1} d_{n}^{m} w_{2 K m+n} p\left(t-n\left(\frac{T_{s y m}}{2}\right)-\right. \\
& \left.m K T_{s y m}-h^{(2 K m+n)} T_{w}\right),
\end{aligned}
$$

where $K$ is a constellation mapper that maps either 1 or 4 bits per symbol to a code word, $d_{n}^{m}$ is the $n^{\text {th }}$ code word component over the $m^{\text {th }}$ symbol, $T_{\text {sym }}$ is the symbol time, $h^{(2 K m+n)}$ is the time-hopping sequence of each waveform position, and $T_{\mathrm{w}}$ is the duration of the waveform. This corresponds to the pulse duration $T_{\mathrm{p}}$ in the single pulse option and $w_{2 K m+n}$ is a scrambling sequence for a burst waveform of duration $T_{\mathrm{w}}$ consisting of $N_{\mathrm{cpb}}$ concatenated and dynamically scrambled short pulses, $p(t)$, each of duration $T_{\mathrm{p}}$. If the single pulse option is applied, $w_{2 K m+n}=1$ in (1).
Both the scrambled pulses in the burst and the time-hopping sequence are defined by a linear feedback shift register which initial polynomial code is the first 14 bits of the Kasami sequence of the used preamble code. The same shift register is used when generating time-hopping sequences for the DBPSK/DQPSK. The difference is that with DBPSK the pulses in the burst are utilized with static scrambling.

After the pulse shaping, the SHR is added and the packet is forwarded to the RF-front-end for the actual transmission.

\section{2) Differentially encoded binary and quadrature PSK}

The bits of the PHR and the PSDU are encoded in a way that the information is contained in the phase change of consecutive PSK symbols.

The spreading of the symbols in the DBPSK/DQPSK is optional and can be utilized for enhancing interference rejection. A 7-bit Barker sequence is used to spread a DBPSK/DQPSK symbol. While enhancing the interference rejection, the spreading reduces the data rate to $1 / 7$ of the original rate.

The transmitted $m^{\text {th }}$ waveform in DBPSK/DQPSK is [13]

$$
x^{m}(t)=\sum_{m=0}^{N} c_{m} w\left(t-m T_{s y m}-h^{(m)} T_{w}\right),
$$

where $N$ represents the number of bits of the PPDU when DBPSK is used. With DQPSK, $N$ is replaced by $N / 2$ and it represents the number of bits of the PHR and the PSDU combined. $c_{\mathrm{m}}$ presents the differential encoding of the transmitted symbol. With DBPSK, $c_{\mathrm{m}}$ carries one bit of information and with DQPSK, two bits. The $w(t)$ is the pulse waveform which in the single pulse option corresponds to $p(t)$ and in the burst pulse option is a burst of $N_{\mathrm{cpb}}$ concatenated and statically scrambled pulses. After the pulse shaping, the SHR is inserted with DBPSK/DQPSK specific ruling.

\section{3) $F M-U W B$}

In the FM-UWB, the PPDU is formed before the modulations and the bits in the PPDU are transmitted with the same procedure, contrary to the IR-UWB. The actual information signal, $b(t)$, is a bipolar Gaussian pulse shape. The subcarrier signal is continuous-phase frequency-shift keying (CP-FSK) as [13]

$$
s(t)=V S\left(2 \pi f_{\text {sub }} t+2 \pi \Delta f_{\text {sub }} \int_{-\infty}^{t} b\left(t^{\prime}\right) d t^{\prime}+\emptyset_{0}\right),
$$

where $V$ is the amplitude and $S(t)$ is the modulating carrier signal, either triangular, sawtooth or sine waveform. The $\Delta f_{\text {sub }}$ $=1 / 2 T_{\text {sym }}$ is the peak frequency deviation and $\emptyset_{0}$ is the initial 
phase of the modulating-carrier signal.

The subcarrier signal $s(t)$ is modulated with wideband frequency modulation in order to create a constant-envelope UWB signal as [13]

$$
V(t)=A \sin \left(2 \pi f_{c} t+2 \pi \Delta f \int_{-\infty}^{t} s\left(t^{\prime}\right) d t^{\prime}\right)
$$

where $\Delta f=K_{0} V$ is the peak frequency deviation and $K_{0}$ is the radio frequency oscillator sensitivity [rad/v]. The FM-UWB is a hybrid modulation targeted for low data rate medical applications.

\section{IEEE 802.15.4-2015 UWB PHYSICAL LAYER}

The IEEE 802.15.4-2015 [12] standard includes several different options for PHY implementation. A compact presentation of the IEEE 802.15.4 revisions is given in [36]. In the latest revision [12], the most significant change concerning IR-UWB is that the radio frequency identification (RFID) UWB is included in the main standard and considered as low rate UWB while the data communication targeted IR-UWB is now high rate UWB.

The UWB PHY is based on impulse radio signaling scheme composed of a combination of burst position modulation (BPM) and binary phase-shift keying (BPSK). The UWB PHY data rates vary from $110 \mathrm{~kb} / \mathrm{s}$ up to $27.24 \mathrm{Mb} / \mathrm{s}$ and the specifications support three different frequency bands of operation; the sub-gigahertz, the low, and the high bands (Fig. 2). In order for a device to be compliant with the standard, the mandatory protocol implementation conformance statement (PICS) must be followed. The mandatory PICS include, e.g., data rate, frequency band, and compliant transmitted pulse.

\section{A. UWB Frame Format}

The UWB PPDU format consists of a SHR preamble, PHR and PSDU, which is the transmission order as well. The SHR preamble is divided into two parts: synchronization (SYNC) field and SFD. There are four different options for the duration of the SHR preamble. This is due to the four different repetitions 16, 64, 1024, or 4096 of a preamble symbol in the SYNC field corresponding to short, default, medium, and long preambles, respectively.

A preamble symbol is constructed based on ternary preamble code with the length of 31 or 127 . The preamble code of length 31 is the mandatory code and the 127 length code is optional. To form one preamble symbol, $L-1$ zeros are inserted between each element of the preamble code. $L$ can have different values depending on the SHR preamble symbol rate. The preamble symbols are transmitted using mandatory rates. These are 0.25 or $1.01 \mathrm{Msymbol} / \mathrm{s}$ for the mandatory code length and 0.98 Msymbols/s for the optional code length.

The length of SFD is 8 or 64 preamble symbols where 8 symbols are used for default and medium data rates and 64 symbols for the optional low data rate of $110 \mathrm{~kb} / \mathrm{s}$. The SFD has its own ternary code from which the SFD preamble symbol is formed with the same procedure as in the SYNC field. The transmission rate of the SFD preamble symbols is equal to the transmission rate of the preamble symbols in the SYNC field.

The PHR consists of 19 bits. It contains information on the used data rate to transmit the PSDU, the frame length, and the preamble duration to name a few. Additionally, six parity check bits are used against channel errors. The parity bits are a simple Hamming block code and they are called single error correction, double error detection (SECDED) bits according to their capabilities. The transmission rate of the PHR is $110 \mathrm{~kb} / \mathrm{s}$ if the PSDU data rate is the same and $850 \mathrm{~kb} / \mathrm{s}$ if the PSDU data rate is equal to or greater than $850 \mathrm{~kb} / \mathrm{s}$. The bits in the PHR are modulated with the BPM-BPSK combination described in detail in the next section.

Fig. 5 presents the schematic diagram of the UWB PHY of the IEEE 802.15.4-2015 and TABLE IV presents the compressed timing parameters related to the UWB PHY, including different FEC rates and their relation to different data rates.

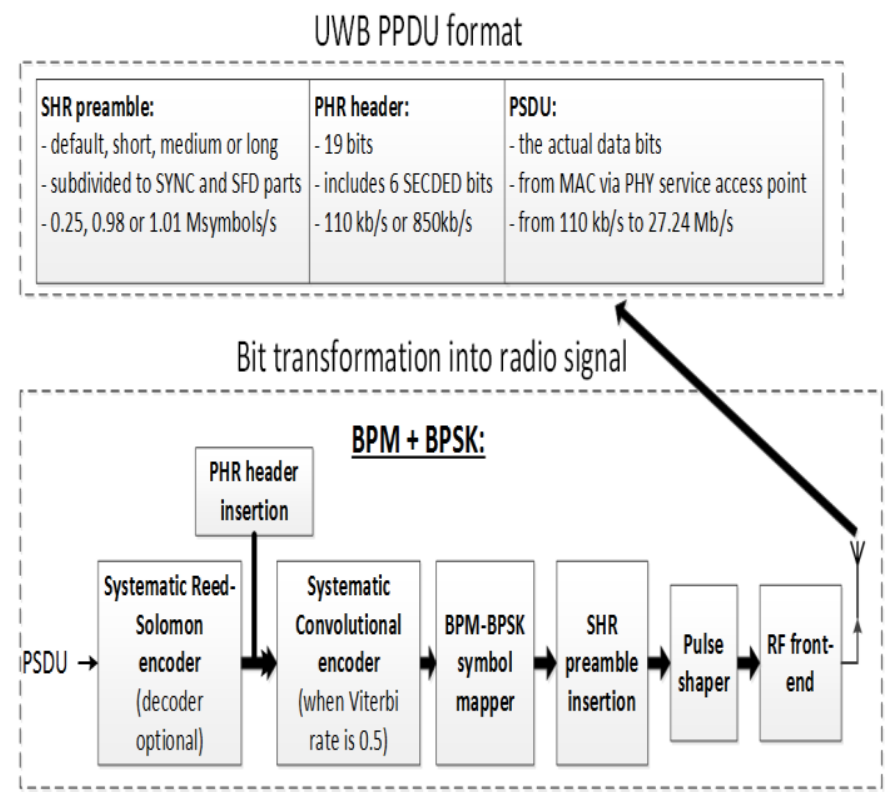

Fig. 5. Schematic diagram of the UWB PHY of the IEEE 802.15.4-2015.

The bits in the PSDU include the actual data bits received from the MAC layer. The first step is the Reed-Solomon (RS) encoding for the PSDU bits. The encoding is systematic $\mathrm{RS}_{6}(63,55)$ code in which multiples of up to 330 bits from the PSDU are converted to 55 RS-symbols. The output of the RSencoder is 63 symbols consisting of 378 bits. The resulting 48 parity bits are systematic which means that a receiver can be implemented without the FEC decoder to achieve some simplicity in the design. In this case, the additional parity bits are simply ignored without achieving any coding gain. The RS-encoded bits are, generally, position modulated, thus a non-coherent receiver is also capable of detecting the encoded bits. Note that the optionality of the channel coding is allowed only to the decoder part at the receiver side. The correction ability of the $\mathrm{RS}_{6}(63,55)$ is at most 4 RS-symbols. It means that there can be one or more erroneous bits in no more than 4 different symbols. 
TABLE IV. IEEE 802.15.4-2015 UWB PHY timing parameters for BPM-BPSK modulations.

\begin{tabular}{|c|c|c|c|c|c|c|c|c|}
\hline$T_{\text {dsym }}(\mathrm{ns})$ & $N_{h o p}$ & $N_{c p b}$ & $T_{\text {burst }}$ (ns) & Data rate (Mbps) & Viterbi rate & RS rate & Overall FEC rate & Preamble code length \\
\hline 8205.13 & 32 & 32 & 264.10 & 0.11 & $\overline{0.5}$ & $\overline{0.87}$ & $\overline{0.44}$ & 31 \\
\hline 1025.64 & 32 & 4 & 8.01 & 0.85 & 0.5 & 0.87 & 0.44 & 31 \\
\hline 512.82 & 32 & 2 & 4.01 & 1.70 & 0.5 & 0.87 & 0.44 & 31 \\
\hline 256.41 & 32 & 1 & 2.00 & 6.81 & 1 & 0.87 & 0.87 & 31 \\
\hline 8205.13 & 8 & 128 & 256.41 & 0.11 & 0.5 & 0.87 & 0.44 & 31 \\
\hline 1025.64 & 8 & 16 & 32.05 & 0.85 & 0.5 & 0.87 & 0.44 & 31 \\
\hline 128.21 & 8 & 2 & 4.01 & 6.81 & 0.5 & 0.87 & 0.44 & 31 \\
\hline 64.10 & 8 & 1 & 2.00 & 27.24 & 1 & 0.87 & 0.87 & 31 \\
\hline 8205.13 & 2 & 512 & 1025.64 & 0.11 & 0.5 & 0.87 & 0.44 & 127 \\
\hline 1025.64 & 2 & 64 & 128.21 & 0.85 & 0.5 & 0.87 & 0.44 & 127 \\
\hline 128.21 & 2 & 8 & 16.03 & 6.81 & 0.5 & 0.87 & 0.44 & 127 \\
\hline 32.05 & 2 & 2 & 4.01 & 27.24 & 0.5 & 0.87 & 0.44 & 127 \\
\hline
\end{tabular}

After the RS-encoding, the PHR is added to the encoded PSDU. These combined bit streams are fed to an optional halfrate systematic convolutional encoder. The half rate, also called as Viterbi rate in the standard [12], means that the convolutional encoder doubles the amount of bits to modulate and to transmit. The systematic aspect is similar as in the RSencoder. A receiver can ignore the additional parity bits if desired. However, the convolutional encoded parity bits are modulated to the phase of the transmitted burst and therefore visible only to a receiver with a coherent detection algorithm. Additionally, for some optional data rates, it is possible to set the Viterbi rate to 1 which disregards the convolutional encoding and thus doubles the actual PHY data rate including the RS-encoded bits.

\section{B. Transformation of Formatted bits into Radio Signals}

After the FEC-encoding, the bit stream is modulated by a combination of BPM-BPSK. The transmitted waveform during the $k^{\text {th }}$ symbol interval is expressed as [12]

$$
\begin{array}{r}
x^{k}(t)=\left[1-2 g_{1}^{(k)}\right] \sum_{n=1}^{N_{c p b}}\left[1-2 s_{n+k N_{c p b}}\right] p(t- \\
\left.g_{0}^{(k)} T_{B P M}-h^{(k)} T_{b u r s t}-n T_{c}\right),
\end{array}
$$

where $g_{0}^{(k)}$ and $g_{1}^{(k)}$ present the bits modulated into the position and the phase of the transmitted burst, respectively. The sequence $s_{n+k N_{c p b}} \in\{0,1\}, n=0,1, \ldots, N_{\mathrm{cpb}}-1$ is the scrambling code during the $k^{\text {th }}$ symbol interval and $h^{(\mathrm{k})} \in\{0$, $\left.N_{\text {hop }}-1\right\}$ is the burst time-hopping position where $N_{\text {hop }}$ defines the number of possible hopping positions during one symbol interval. The $p(t)$ is the mandatory pulse shape at the antenna input, $T_{\mathrm{BPM}}$ represents the position modulation delay, i.e., a half symbol length, $T_{\text {burst }}$ is the burst length, and $T_{\mathrm{c}}$ is the duration of one pulse.

The shape of the mandatory pulse is not defined but the cross-correlation with the reference pulse is the restrictive property. The $p(t)$ needs to have a normalized crosscorrelation magnitude with a reference pulse greater than 0.8 . It needs also to comply with the standard defined timing parameters and to fulfill the spectral mask requirements of the standard and the regulatory spectral mask where applicable.

The UWB symbol structure of the standard is presented in Fig. 6. The transmitted burst defined in Eq. (5) is placed in one of the burst hopping positions during the symbol interval.
In total, there are $4 N_{\text {hop }}$ burst positions in one symbol but only one is occupied as the remaining are left empty for improving multiuser interference rejection. The actual burst positions that can be used are located in the first and in the third quarter of the symbol. The second and the fourth quarters are guard intervals against inter-symbol-interference (ISI) and at the same time providing multiuser interference rejection. When transmitting bit ' 0 ', the burst is placed in the first quarter and transmitting bit ' 1 ', in the third quarter, thus reducing the available burst positions to $N_{\text {hop }}$.

Both the scrambling code and the time-hopping sequence are computed by a common linear feedback shift register. As the time varying hopping code provides multiuser interference suppression capability for different types of receivers, the scrambling code for polarity changes of the single pulses in the burst is intended for coherent receivers only. Additionally, the scrambled burst is providing spectral smoothening of the transmitted waveform.

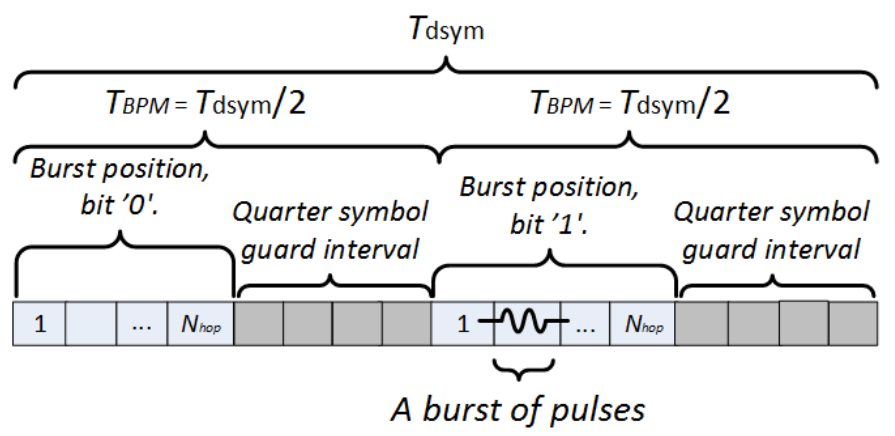

Fig. 6. UWB symbol structure of the IEEE 802.15.4-2015.

The UWB PHY includes also some optional features. These include support for ranging and optional pulse shapes, in addition to the channel decoding at the receiver side as described earlier. The ranging is based on a two-way ranging protocol between two ranging capable devices therefore allowing the ranging measurement without a common time reference. The ranging is supported only by the UWB PHY of the IEEE 802.15.4-2015 [12].

The optional pulse shapes are chirp pulses, continuous spectrum pulses, and linear combination of pulses. However, the use of these is limited to non-beacon frames only and the modulation and timing specifications will remain as defined for the mandatory pulse. The optional pulse shapes are based on the $p(t)$ but with additional operations. In the chirp pulses, 
there is multiplication with an exponential function producing either up- or downslope of frequency of the mandatory pulse. In the continuous spectrum pulses, there is a group delay generated for the mandatory pulse by passing it through an allpass filter. The linear combination of pulses, on the other hand, is formed by summing 4 weighted and delayed mandatory pulses.

\section{COMPACT COMPARISON OF THE STANDARDS}

A comparison of the UWB PHYs of the two standards reveals that the main differences relate to the used modulation methods and to the channel coding. There are two modulation methods (BPM-BPSK) specified in the WPAN standard [12]. However, they are used as a combination of both or only the BPM. In the WBAN standard [13], there are three different modulation options (OOK, DPSK, FM-UWB) which are used independently of the others.

The channel encoding in the IEEE 802.15.4-2015 UWB PHY layer is utilized based on RS and convolutional encoding which both are redundant and systematic. Thus, a receiver can ignore the coding if desired. In the IEEE 802.15.6-2012, the channel errors are reduced by a combination of $\mathrm{BCH}$-codes, with different possible coding strengths, and bit interleaving. Other differences include ranging and RFID, which are not included in the WBAN standard, but are supported by the WPAN standard. Regarding the rather small differences in the data rates and frequency utilization, TABLE I and Fig. 2 present these in detail, respectively. Furthermore, TABLE V summarizes the UWB PHY comparison of the two standards.

The main similarities between the two UWB PHYs relate to IR-UWB waveform, the symbol structure, and the frequency band allocations. The transmitted waveform in the burst mode is constructed in a similar manner, the symbol structure is almost identical, and the defined frequency bands are overlapping for the most parts (Fig. 2). Even the detection of signals can be made according to the same principle. This requires that in the WBAN transmission of an OOK modulated signal, variable $K$ is set to 1 . Both standards enable also the use of chirp pulse signal, even though in the WPAN standard, its use is limited to non-beacon frames only.

TABLE V. A comparison of the PHYs of the two standards.

\begin{tabular}{|c|c|c|}
\hline Feature & IEEE 802.15.6-2012 & IEEE 802.15.4-2015 \\
\hline $\begin{array}{c}\text { Number of different } \\
\text { PHYs }\end{array}$ & 3 & 18 \\
\hline Ranging & No & Yes \\
\hline RFID & No & Yes \\
\hline $\begin{array}{l}\text { Max. UWB PHY } \\
\text { data rate }\end{array}$ & $15.6 \mathrm{MHz}$ & $27.24 \mathrm{MHz}$ \\
\hline UWB modulations & $\begin{array}{c}\text { OOK, } \\
\text { DBPSK/DQPSK, } \\
\text { FM-UWB }\end{array}$ & BPM-BPSK \\
\hline Encoding & $\overline{\mathrm{BCH}}$ & RS, Convolutional \\
\hline Bit interleaving & Yes & No \\
\hline $\begin{array}{c}\text { No. of UWB } \\
\text { frequency channels }\end{array}$ & 11 & 16 \\
\hline Chirp pulses & Yes & Yes \\
\hline UWB duty cycle & $3.125 \%$ & $0.8 \%, 3.1 \%$ or $12.5 \%$ \\
\hline
\end{tabular}

\section{LITERATURE REVIEW}

This section presents an overview of the work performed in the UWB studies related to both of the IEEE standards. The main focus is on PHY layer aspects and the time scale of the presented research is 2007-2015. A recent UWB survey can be found in [37]. Despite presenting quite a long historical view covering twenty years, it focuses on hardware development and challenges over the period, particularly that of the MBUWB. Another survey article is [38]. Besides presenting different UWB technologies, its main focus is set on the presentation of existing commercial devices and a platform developed in a European Commission driven project.

To the best of our knowledge, these two, [37] and [38], are the only surveys with the main focus on UWB from the past ten years. However, neither of these surveys presents IR-UWB in an in-depth manner nor do they consider the IR-UWB related standards and the research related to these, which is the main contribution of this survey.

A few statistics collected from the IEEE Xplore digital library are presented in Fig. 7 concerning publications between the years 2000-2015 concentrating on UWB and the two standards. The red bars present the annual number of published publications including the keyword/abbreviation 'UWB'. The square marked curve and the circle marked curves represent the number of publications in each year that were found with the search words ' 802.15 .4 ' and '802.15.6', respectively.

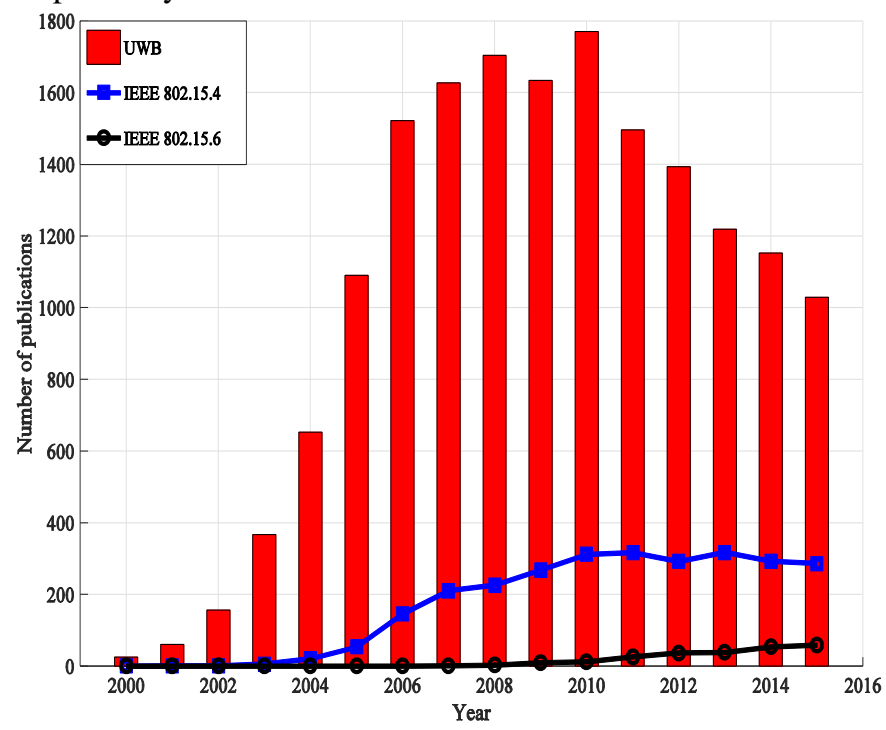

Fig. 7. The annual number of publications with the selected keywords in the IEEE Xplore digital library.

Related to the IEEE 802.15.4-2015 studies, the majority of the articles address some other topic than UWB specifications, i.e. ZigBee or other PHYs that are also included in the standard. Considering the IEEE 802.15.6-2012 studies, UWB is the most popular topic out of the modest number of publications and of the three PHY layers defined by it. Additionally, the majority of the references presented in this survey are listed and categorized in TABLE VI including the articles that will be discussed next. 
TABLE VI. Categorization of references.

\begin{tabular}{|l|l|}
\hline Topic & Reference number \\
\hline \hline UWB regulatory & {$[5]-[7]$} \\
\hline UWB standards & {$[12],[13]$} \\
\hline $\begin{array}{l}\text { WPAN and WBAN technology } \\
\text { comparisons }\end{array}$ & {$[23],[26],[33]-[35]$} \\
\hline UWB surveys & {$[37],[38]$} \\
\hline Implemented UWB systems & {$[39]-[61]$} \\
\hline Ranging and positioning & {$[44],[50],[62]-[70]$} \\
\hline Medical and healthcare & {$[71]-[81]$} \\
\hline $\begin{array}{l}\text { Interference and coexistence } \\
\text { studies }\end{array}$ & {$[82]-[90]$} \\
\hline Synchronization & {$[91]-[93],[95]$} \\
\hline
\end{tabular}

Regarding the next sections, the division of topics is based on general frameworks of research. The presentation order follows the popularity of the topic measured by the number of articles found and presented here. The channel and propagation topics and the MAC studies have been excluded from the focus of this article as the main target is on UWB PHY layer related to the WPAN and WBAN standards, as stated in the beginning of this article.

\section{A. Implemented Systems [39] - [61]}

The majority of the articles concerning hardware implementation can be roughly sub-divided into prototype studies [40]-[50], pulse generation [51]-[55], and power consumption/efficiency studies [56]- [59].

A good starting point is the article [39], which briefly reviews and summarizes UWB transceiver challenges until 2010. It also represents some receiver schemes taking into account low-complexity requirement and the existing WPAN standard as the WBAN standard was not yet published.

The work in [40] introduces one of the first published WPAN standard compliant prototypes. The authors demonstrate a system with non-coherent receivers capable of both data transmission and ranging with $15 \mathrm{~cm}$ accuracy up to $10 \mathrm{~m}$ distance using the authors' own designed algorithm. Another early prototype related to the WPAN is tested and evaluated in [41]. It includes a standard compliant receiver along with a new system setup analysis under several different channel conditions. Also a standard compliant IR-UWB circuit of a digitally controlled oscillator is presented in [42].

One of the first WBAN standard compliant prototypes is presented in [43]. Besides the UWB PHY with DBPSK, the system model includes the MAC layer which is also based on the WBAN standard specifications.

In [44], the authors present a chipset transceiver compliant with the both WBAN and WPAN standard specifications, including ranging. Moreover, the operating frequency is between 7 and $10 \mathrm{GHz}$ making it compatible with worldwide regulatory definitions.

A comparative study is reported in [45] which compares IRUWB, FM-UWB, and a commercially available narrow band radio in terms of power consumption, reliability, and latency. The UWB specifications were partially taken from the WPAN standard and also from the WBAN draft standard.
In [46] the authors evaluate a dual radio architecture for the use in low-power WBANs. In their proposal, a transmit-only IR-UWB radio in a node is equipped with a narrowband transceiver for exchanging information and reducing the power consumption as the IR-UWB receiver consumes more power than a narrowband receiver. A similar idea is demonstrated in [47] concerning high-speed video transfer but now with OFDM-UWB for transmitting data and ZigBee for sending control information back to nodes. Other dual approaches are presented in [48] and in [49]. Both articles evaluate a receiver architecture which is a combination of coherent and non-coherent demodulations. The non-coherent one is used for fast synchronization while the coherent demodulation structure is used for data detection. The initial idea, however, is thoroughly presented and analyzed in [50] where it is also used for accurate ranging, in addition to data transmission.

Regarding pulse design, the authors of [51] investigate pulse generations that achieve center frequencies up to $9 \mathrm{GHz}$ while offering less than $-85 \mathrm{dBm} / \mathrm{MHz}$ signal strengths below $6 \mathrm{GHz}$. In addition to the FCC limits, the generated pulses are compliant with Japanese and European UWB regulations as well. Another article focusing on pulse generation is [52]. With both WPAN and WBAN standards compatible pulse design, the article presents a comparison of existing designs from the point of view of pulse energy consumption.

Related to the FCC spectral mask and the IEEE 802.15.42015 pulse requirements, in [53] an algorithm is studied and proposed to generate orthogonal pulses to fulfil the above mentioned definitions. One of the latest articles considering pulse generation is [54]. The article presents a differential voltage controlled oscillator which can cover the frequency bands defined by both of the standards. The oscillator achieves fast startups and stops without the need for a mixer and it can also be shut down between consecutive pulse transmissions to save power. Considering the optional chaotic pulse waveform of the WPAN standard, a compliant transceiver system is studied in [55].

Power consumption issues and implementation design of a WPAN standard compatible IR-UWB transceiver system is studied in [56]. Detailed power consumption results of different blocks of the transceiver are provided and some of the most critical steps of the hardware design in order to achieve a power efficient system are considered. The same authors continued their studies later on in [57] with the main focus on synchronization and detection performance.

An in-depth analytical and also experimental approach is presented in [58]. Besides presenting WPAN standard definitions, the authors provide analytical results of a method to estimate the peak power of IR-UWB pulse. The method is also verified by an experimental setup. The target and the importance of accurate peak power measurement is related to the maximum achievable distance of the radio link as the peak power limitations apply to UWB signal transmission in the global regulations.

A recent article [59] proposes and analyzes power efficiency of an IR-UWB transmitter with OOK. Additionally, 
they compare also its power efficiency and a few other metrics to other existing architectures found in the literature.

Even living animals are reported to be used in real measurement campaigns. In both [60] and [61], the authors use a living pig to perform implant communication measurements. In the former, MB-OFDM is used and the in the latter, IR-UWB. Despite strong signal attenuation inside the pig, they are able to achieve reasonable results in terms of $\mathrm{BER}$ and data rate.

\section{B. Ranging and Positioning [44], [50], [62] - [70]}

Articles listed in the category of "ranging and positioning" consider the IEEE 802.15.4-2015, due to the fact that in the IEEE 802.15.6-2012 the ranging protocol is not defined.

Article [62] is one of the first journals to consider the initial release of the WPAN standard and its' ranging definitions. The work includes a thorough literature review as well as presentations of different positioning estimation techniques, e.g., time-of-arrival and received signal strength indication. The authors consider also signal design and receiver structures suitable for the positioning and ranging. Different IR-UWB receiver structures are analyzed and compared also in [63] from a device complexity vs. location accuracy point of view.

Security of the IEEE 802.15.4-2015 ranging protocol is studied in [64] with a protocol proposition to improve the security of the positioning.

In [65], the authors analyze and demonstrate a timing counter management scheme shown to improve the ranging protocol defined by the standard which is prone to clock frequency offset.

Ranging accuracy measurements of a positioning system based on the IEEE 802.15.4-2015 are performed in [66]. The achieved accuracy is reported to be below $10 \mathrm{~cm}$ in both indoor and outdoor measurements.

In [67] positioning is studied from power control point of view demonstrating a new method improving ranging performance in realistic environments.

In [68] the authors combine UWB ranging and interference studies. They provide a presentation of the existing time-ofarrival estimation algorithms and then analytically study and present their own solution which is shown to suppress the interfering multiuser signals.

A double-quadrature architecture is also used for ranging in [50], in addition to data transmission. A thorough analysis of the architecture and a comparison of the previous published UWB ranging receivers are also provided in [50] taking into account power consumption, ranging accuracy, and sensitivity.

One of the latest articles with an in-depth analytical perspective is [69]. The article provides an extensive literature survey together with new algorithms that are simulated with the IEEE 802.15.4-2015 compliant parameters. Another newly published article is [70], which studies and proposes a method for increasing security in a network. Both IR-UWB and ZigBee based systems are compared.

\section{Medical and Healthcare [71] - [81]}

The article in [71] is among the first articles to consider the WPAN standard for telemedicine purposes. It presents throughput requirements for different on-body measurements while taking into account the WPAN standard specifications with link budget calculations. Another one can be found from [72], where an overall picture of the use of UWB based WBANs is presented for medical applications together with other existing wireless technologies.

Both [73] and [74] present simulation results of different receiver structures including different rake and ED receivers that are capable of detecting the WPAN standard defined signal model. Additionally, channel models based on hospital measurements, are used in the simulations.

A review and a presentation of both IR-UWB and FMUWB techniques related to the WBAN standard is presented in [75] providing different perspectives of biomedical applications and design challenges.

A system for electrocardiograph measurement based on the WPAN standard is proposed in [76]. Interestingly, transmit only type IR-UWB radio is used and it is studied more thoroughly in [77].

In [78], the authors propose ideas of an architecture for medical BAN which connects both in-body and on-body sensors and is utilized by both IR-UWB and MB-UWB techniques. In [79], partially by the same authors, their medical BAN architecture is extended by adding cognitive radio features on it.

Evaluations of energy detector receivers based on the WBAN standard is presented in [80] for symptom detection of Parkinson's disease. Another WBAN standard based article is [81], where a WBAN PHY compatible transceiver system with hospital environment channel models is used for comparing receivers with differential detection.

\section{Interference and Coexistence [82] - [90]}

The article [82] addresses different IR-UWB receiver structures and their tolerances against multiple access interference. Besides providing a literature review, the authors compare and analyze existing approaches and evaluate performance characteristics of such receivers to their proposals with a joint optimization of demodulation and decoding approach.

Interference mitigation protocols are studied in [83] and a 4-step interference mitigation protocol is also proposed. It takes into account the WBAN standard traffic priorities while increasing spatial reuse between coexisting WBANs by allocating orthogonal channels for interfering nodes and allowing simultaneous transmissions between nodes with low interference level.

IEEE 802.15.6-2012 IR-UWB receiver performances against FM-UWB and IEEE 802.15.4-2015 interferers is investigated in [84]. In [85], the authors investigate UWB coexistence from multiple angles. The main focus is on a MBUWB system and its performance when other systems, i.e., Wi-Fi and ZigBee, operate close to and partially on the same bands as the inspected system. Additionally, both IR-UWB 
and MB-UWB system performances are evaluated when they interfere with one another.

An interference study is performed in [86] comparing two types of receivers, which are capable of detecting both the traditional IR-signaling as well as the chirp pulse signal, defined also in the WBAN standard. Interfering sources include both FM-UWB and IR-UWB signals.

In [87], the authors investigate concatenated coding scheme defined in the WPAN standard and the performance characteristics of it under fading channels and with multiuser interference. The semi-analytical model is verified by simulations.

The article [88] focuses on ED receivers and their performance improvement by mitigating interference with statistical interference modeling. Several receiver types with different mitigation approaches are also compared. Another article considering ED receivers is [89], where the performance of receivers based on the IEEE 802.15.6-2012 are evaluated in the presence of multiuser interference in realistic channel models.

Unconventional surroundings are presented in [90] where the authors consider the WPAN standard for intra-spacecraft communications. They use channel models based on measurements inside a spacecraft when evaluating the performance of ED receivers.

\section{E. Various Other Approaches [91] - [102]}

There are several articles that fall outside the topics discussed above. Regarding the other topics, synchronization is one of the most popular ones related to IR-UWB.

An overview of some popular existing synchronization algorithms can be found in [91] together with comparative results and discussion. In [92], the authors evaluate the performance of two synchronization procedures of an IRUWB system in industrial environment based on the IEEE 802.15.4-2015 PHY specifications. The research is further continued in [93]. Also related to an industrial environment, the article [94] evaluates the WPAN standard suitability and analyzes its coverage range in an industrial context. In another synchronization focused article [95], the authors propose a low-complexity architecture with a WBAN standard compliant synchronization algorithm. The article presents also results of the implemented system based on non-coherent receivers.

Related to RFID-UWB, the article [96] proposes a combined network of the IEEE 802.11 and the IEEE 802.15.4 standards to be used for item monitoring and tracking. In the proposal, the IEEE 802.11 is used for the main communications link between the hub and the nodes as the IRUWB based radio is used for RFID. An extensive analysis and literature survey regarding the RFID-UWB is found in [97].

Another combination of technologies is found in [98], where an IR-UWB radar and ZigBee radio is studied for cardiopulmonary monitoring. The UWB radar detects the heart and breath rates and the measurement data is transmitted through an IEEE 802.15.4 based ZigBee radio.
Energy-efficiency regarding IR-UWB is presented in [99]. The authors propose and analyze a link adaptation scheme for non-coherent receivers that can adapt the number of pulses per symbol to the channel conditions to maximize energy efficiency. This is, according to the authors, applicable to any non-coherent IR-UWB system. In [100], energy efficiency is studied also, now from the cross-layer optimization view point. The proposed model is based on the WBAN standard specifications by enabling joint code rate and packet length optimization.

Beamforming and multiple input and multiple output (MIMO) studies have also been performed over the years. Article [101] presents a chirp signal beamformer and also compares it to existing methods. Chirp pulses can be used in both of the IEEE standards. Regarding MIMO antennas in UWB, an in-depth analytical work can be found in [102] together with an extensive literature survey of the topic.

\section{CONSIDERATIONS FOR IMPROVING DATA RATES OF IEEE 802.15.6-2012 AND IEEE 802.15.4-2015}

The data rate considerations of the two standards are performed mainly for the future demands of sensor networks. At the moment, these two standards are targeted for lowcomplexity and for modest/low data rate applications. Yet, the general development of wireless communications has shown that the data rate demands tend to increase over time. Therefore, we present some options, focusing on modulation methods and PHY symbol structure, on how to increase the currently specified data rates. We realize that any, even minor, change to the existing standards can be difficult. Also, the increase in data rate often adds the system complexity as well. These two issues are left unaddressed here as we present ideas found from the literature and the possibilities they provide if utilized into the existing standards and their future revisions.

Regarding the PHY data rate increase/scalability of the two IEEE standards, there are several different options. The most obvious one is already utilized in the standards by allowing different symbol lengths thus enabling the increase of the symbol transmissions during a time interval. The effect of the various symbols lengths on the PHY data rate scale is visible in TABLE II and TABLE III for the IEEE 802.15.6-2012 and in TABLE IV for the IEEE 802.15.4-2015.

In the WBAN standard, the data rate scales from 0.487 Mbps to $15.60 \mathrm{Mbps}$ and in the WPAN standard, from 0.11 Mbps to $13.62 \mathrm{Mbps}$ by simply changing the duration of one PHY symbol. Note that the maximum PHY data rate for the WPAN standard is 27.24 Mbps but it is achieved only if the convolutional coding is disregarded and both the position and the phase of the burst are carrying data bits, thus a combination of binary BPM-BPSK is used.

Many other examples of combinations of IR-UWB modulations can be found in the literature. The studied combinations include, at least, pulse shape modulation (PSM) and PPM in [103] which actually includes also pulse amplitude modulation (PAM) as there is a possibility to scale the transmissions up to 3-bits per symbol. Additionally, a PAM-PSM combination is presented in [104] where the 
authors study also PPM and extending these to $M$-ary modulations.

From non-coherent receivers and the standardized symbol structure point of view, extending PPM to $M$-ary is not increasing the data rate as there are limitations from the propagation delay of the signal. As pointed out in [29], the typical UWB channel delay spread varies between 10 and 50 ns. Therefore, the shortest symbol lengths defined in the standards provide already the highest data rates for the PPM in a realistic channel and an extension to $M$-ary would not increase it. Considering $M$-ary PSK-modulation in IR-UWB, the extension is possible with the chirp pulse waveform as the generation and detection of short pulse phase is feasible only with a binary signal.

Combining OOK and PSM is studied in [105] including $M$ ary modulations of PSM. Another modulation "combination" is proposed in [106], called binary pulse shape frequency shift keying. It is a modification of pulse shape modulation in which instead of the traditional orthogonal pulses, utilizes ones that are located in different frequencies.

A novel approach to increase the data rate of transmitted reference signaling and also to include channel coding into the modulation is presented in [107]. The authors present a hybrid combination of PPM-BPSK and transmitted reference signaling with a 2-bit mapping scheme which have better BER performance than the PPM-BPSK defined by the IEEE 802.15.4-2015. Another hybrid modulation idea is presented in [108], where improvement to the existing PPM-BPSK modulation of the WPAN standard is proposed. It is done by a phase-directed position estimation algorithm. The algorithm is constructed by bit mapping, where the position of the burst in the next symbol can be estimated from the phase information of the previous symbol.

The authors of [109] propose an idea that instead of the PPM modulation and the required symbol structure, any other IR modulations could be used instead. In this manner, the same 2 bits per symbol, when using PPM-BPSK combination, could be transmitted but only with the use of one modulation type. Compared to non-coherent receivers, the PHY data rate is doubled and the whole network level throughput is improved by reducing the number of transmitted symbols [110].

Basically, combining binary modulations in IR-UWB adds a bit per modulation per symbol into the transmission of the signal. Any of the presented modulation combination examples can be used for increasing data rate in IR-UWB communications, especially if $M$-ary signaling is included. However, as mentioned earlier, the complexity increases as well but, in theory, this is one way to increase the data rate of the current standards. Also, including simpler/modified coding schemes to the modulation, the detection performance can be improved as was the case with hybrid modulation studies.

Related to both the data rate improvement and the comparisons of the two standards in Section VI, we have proposed in our earlier work, e.g., [81] and [109] that it would be beneficial to allow the use of different modulations in the standards or even combine the two existing IR-UWB PHY layers. The signal generation and transmission is very similar in the IR-UWB, despite of the modulation used. Therefore, increased compatibility between the standards' PHYs would help to build a radio chip that would be compliant for both. This would not require major changes even if implemented with the current specifications of the two standards. Additionally, a pool of different modulations would increase the scalability of a sensor network to adapt for different demands, from increased data rate to maximizing battery life.

\section{CONCLUSIONS}

This article presented an updated survey on IR-UWB communications from 2007-2015. In addition to the survey, we presented the current global regulatory spectrum allocation of UWB. We explored also the PHY specifications of the two existing IEEE standards including IR-UWB. The majority of the surveyed literature is categorized in TABLE VI including also other short-range technologies. Additionally, TABLE VII lists all the different acronyms mentioned in this article in alphabetical order.

A view to the future, we believe that UWB will be a part of the future wireless communications systems and protocols, at least because of the definition of minimum $500 \mathrm{MHz}$ of bandwidth. Especially at the $60 \mathrm{GHz}$ bands and above due to the available spectrum, the minimum bandwidth for a signal to be UWB is fairly easily achieved with multiple subcarriers.

Another aspect is the currently studied and standardized UWB and its role in the future. ZigBee and particularly Bluetooth are the dominating short-range communication technologies at the moment and their features, such as power consumption, have developed over the years. However, we believe that IR-UWB will also have a role in the future in special applications areas and not only due to the definitions fulfillment. Firstly, neither ZigBee nor Bluetooth provide a power consumption level for a sensor device that could last for years. Secondly, the positioning accuracy of the two abovementioned short-range communications technologies is rather imprecise. Thirdly, the available spectrum for the currently used technologies, including mobile technologies, is limited appearing in interference issues.

IR-UWB is capable of meeting these requirements and claiming at least part of the huge potential that was loaded on it in the late 90 's and the early 2000's. IoT applications measuring condition of a human or a machine do not necessary need high data rates but very long battery life. For a future RFID system, for example, the high accuracy of ranging resolution has been expected to be provided and for this, IRUWB is more than well capable. From spectrum accessibility point of view, the globally available UWB spectrum would be a valuable addition to the current spectrum usage and management. One option for these future demands can be a dual radio chip composed of both WPAN and WBAN standards based solutions. Moreover, the low EIRP level of UWB devices results in low interference to the existing communications systems. 
TABLE VII. List of the used acronyms.

\begin{tabular}{|c|c|}
\hline Acronym & Meaning \\
\hline $\mathrm{BCH}$ & "Bose-Chaudhuri-Hocquenghem \\
\hline BPM & burst position modulation \\
\hline BPSK & binary phase-shift keying \\
\hline CP-BFSK & continuous phase binary frequency-shift keying \\
\hline DAA & detect and avoid \\
\hline DARPA & Defense Advanced Research Project Agency \\
\hline DBPSK & differential binary phase-shift keying \\
\hline DQPSK & differential quadrature phase-shift-keying \\
\hline EIRP & equivalent isotropic radiated power \\
\hline FCC & Federal Communication Commission \\
\hline FEC & forward error correction \\
\hline FM & frequency modulation \\
\hline HBC & human body communication \\
\hline high QoS & high-quality of service \\
\hline IEEE & Institute of Electrical and Electronics Engineers \\
\hline IoT & Internet-of-Things \\
\hline IR & impulse radio \\
\hline ISI & inter-symbol-interference \\
\hline LDC & low duty cycle \\
\hline MAC & medium access control \\
\hline MB & multiband \\
\hline MIMO & multiple input, multiple output \\
\hline MPDU & MAC protocol data unit \\
\hline NFC & near field communication \\
\hline OFDM & orthogonal frequency division modulation \\
\hline OOK & on-off keying \\
\hline OSD & Office of the Secretary of the Defense \\
\hline PAM & pulse amplitude modulation \\
\hline PHR & physical layer header \\
\hline PHY & physical layer \\
\hline PICS & protocol implementation conformance statement \\
\hline PPDU & physical layer protocol data unit \\
\hline PPM & pulse position modulation \\
\hline PSDU & physical layer service data unit \\
\hline PSM & pulse shape modulation \\
\hline RFID & radio frequency identification \\
\hline $\mathrm{RS}$ & Reed-Solomon \\
\hline SECDED & single error correction, double error detection \\
\hline SFD & start-of-frame delimiter \\
\hline SHR & synchronization header \\
\hline SYNC & synchronization \\
\hline UWB & ultra wideband \\
\hline WBAN & wireless body area network \\
\hline WPAN & wireless personal area network \\
\hline WSN & wireless sensor network \\
\hline
\end{tabular}

\section{ACKNOWLEDGMENTS}

The authors would like to thank the anonymous reviewers for their valuable comments for improving the manuscript. Additionally, Mrs. Hanna Saarela is greatly acknowledged for her effort while improving the readability of the manuscript.

\section{REFERENCES}

[1] OSD/DARPA Ultra-Wideband Radar Review Panel, "Assessment of Ultra-Wideband (UWB) Technology," Office of the Secretary of Defense, Defense Advanced Research Project Agency, Columbus, OH, R-6280, July, 1990.

[2] T. W. Barrett, "History of UltraWideBand (UWB) Radar \& Communications: Pioneers and Innovators," in Progress in
Electromagnetics Symposium, (PIERS 2000), Cambridge, MA, July, 2000.

[3] R. A. Scholtz, "Multiple access with time-hopping impulse modulation," in MILCOM 93, Boston, MA, USA, 11-14 Oct. 1993, vol. 2, pp. 447450.

[4] M. Z. Win and R. A. Scholtz, "Impulse radio: How it works," IEEE Communications Letters, vol. 2, pp. 36-38, Feb. 1998.

[5] Federal Communications Commission (2002) Revision of Part 15 of the Commission's Rules Regarding Ultra-Wideband Transmission Systems, First Report and Order, 118 p. ET Docket, FCC 02-48, [Online], Available: www.fcc.gov, http://fjallfoss.fcc.gov/edocs_public/attachmatch/FCC-02-48A1.pdf.

[6] Electronic Communications Committee (2006) ECC Decision of 24 March 2006 on the Harmonic Conditions for Devices Using UltraWideband (UWB) Technology in Bands Below $10.6 \mathrm{GHz}$. ECC/DEC/(06)04, amended 6 July 2007, amended 9 December 2011, 15 p.

[7] Association of Radio Industries and Businesses (2015) UWB (UltraWideband) Radio Systems. STD-T91, Version 2.0, English translation, March 17, 2015, 75 p.

[8] Integrated Project - EUWB (2011). "Worldwide regulation and standardization overview," [Online], Available: www.euwb.eu/deliverables/EUWB_D9.1_v1.0_2008-09-15.pdf.

[9] K. Mandke, H. Nam, L. Yerramneni, C. Zuniga and T. Rappaport, "The Evolution of Ultra Wide Band Radio for Wireless Personal Area Networks," High Frequency Electronics, vol. 2, No. 5, pp. 22-32, Sep. 2003.

[10] [Online]. Available: http://www.ieee802.org/15/pub/TG3a.html

[11] High Rate Ultra Wideband PHY and MAC Standard, ECMA-368 standard, $3^{\text {rd }}$ Edition, Dec. 2008.

[12] IEEE Standard for Low-Rate Wireless Personal Area Networks (WPANs)", in IEEE Std 802.15.4-2015 (Revision of IEEE Std 802.15.42011), pp. 1-709, April. 222016.

[13] IEEE Standard for Local and metropolitan area networks - Part 15.6: Wireless Body Area Networks", in IEEE Std 802.15.6-2012, pp.1-271, Feb. 292012.

[14] A.F. Molisch, P. Orlik, Z. Sahinoglu, J. Zhang, "UWB-based sensor networks and the IEEE 802.15.4a standard - a tutorial," in $1^{\text {st }}$ International Conference on Communications and Networking in China, (ChinaCom '06), Beijing, China, pp. 1-6, 25-27 Oct. 2006.

[15] L. De Nardis and M-G. Di Benedetto, "Overview of the IEEE 802.154/4a standards for low data rate Wireless Personal Data Networks," in 4th Workshop on Positioning, Navigation and Communication, WPNC '07. Hannover, Germany, 22-27 March 2007, pp. 285-289.

[16] J. Zhang, P.V. Orlik, Z. Sahinoglu, A.F. Molisch and P. Kinney, "UWB Systems for Wireless Sensor Networks," Proceedings of the IEEE, vol. 97, no. 2, pp. 313-331, Feb. 2009.

[17] E. Karapistoli and F-N. Pavlidou, "An Overview of the IEEE 802.15.4a Standard," IEEE Communications Magazine, vol. 48, no. 1, pp. 47-53, Jan. 2010.

[18] K.S. Kwak, S. Ullah and N. Ullah, "An Overview of IEEE 802.15.6 Standard," in $3^{\text {rd }}$ International. Symposium on Applied Sciences in Biomedical and Communication Technologies, (ISABEL 2010), Rome, Italy, pp. 1-6, 7-10 Nov. 2010.

[19] M. Li and M. Zhuang, "An Overview of Physical Layers on Wireless Body Area Networks," in International Conference on AntiCounterfeiting, Security and Identification (ASID 2012), Taipei, Taiwan, pp.1-5, 24-26 Aug. 2012.

[20] S. Ullah, M. Mohaisen, and M. A. Alnuem, "A Review of IEEE 802.15.6 MAC, PHY, and Security Specifications," International Journal of Distributed Sensor Networks, vol. 2013, Article ID 950704, 12 pages, 2013. doi:10.1155/2013/950704.

[21] M. Hernandez and L. Mucchi, "Body Area Networks using IEEE 802.15.6," London, UK: Elsevier Ltd., 2014, pp. 1-44.

[22] B. Latre, B. Braem, I. Moerman, C. Blondiaaand P. Demeester, "A survey on wireless body area networks," Wireless Network, vol. 17, pp. 1-18, Jan. 2011.

[23] S. Movassaghi, M. Abolhasan, J. Lipman, D. Smith and A. Jamalipour, "Wireless Body Area Networks: A Survey," IEEE Communications Surveys \& Tutorials, vol. 16, no. 3, pp. 1658-1686, 2014.

[24] R. Cavallari, F. Martelli, R. Rosini, C. Buratti and R. Verdone, "A Survey on Wireless Body Area Networks: Technologies and Design Challenges," IEEE Communications Surveys \& Tutorials, vol. 16, no. 3, pp. 1635-1657, 2014. 
[25] S. Gonzalez-Valenzuela, X. Liang, H. Cao, M. Chen and V.C. Leung, "Body Area Networks," in Autonomous Sensor Networks: Collective Sensing Strategies for Analytical Purposes, Verlag-Berlin-Heidelberg, Springer, 2013, pp. 17-37.

[26] M. R. Yuce, "Implementation of wireless body area networks for healthcare systems," Sensors and Actuators A: Physical, vol. 162, issue 1, pp. 116-129, July 2010.

[27] D.B. Smith, D. Miniutti, T.A. Lamahewa, L.W. Hanlen, "Propagation Models for Body-Area Networks: A Survey and New Outlook," IEEE Antennas and Propagation Magazine, vol. 55, no. 5, pp. 97-117, Oct. 2013.

[28] M. Seyedi, B. Kibret, D. T. H. Lai, and M. Faulkner, "A survey on intrabody communications for body area network applications," IEEE Transactions on Biomedical Engineering, vol. 60, no. 8, pp. 2067-2079, Aug. 2013.

[29] A.F. Molisch, "Ultra-Wide-Band Propagation Channels," Proceedings of the IEEE, vol. 97, no. 2, pp. 353-371, Feb. 2009.

[30] E. Karapistoli, D. G. Stratogiannis, G. I. Tsiropoulos and F-N. Pavlidou, "MAC Protocols for Ultra-Wideband AdHoc and Sensor Networking: A Survey," in $4^{\text {th }}$ International Congress on Ultra Modern Telecommunications and Control Systems and Workshops, (ICUMT 2012), St. Petersburg, Russia, pp. 834-841, 3-5 Oct. 2012.

[31] K.M.S. Thotahewa, J-M- Redoute and M.R. Yuce, "Medium Access Control (MAC) Protocols for Ultra-Wideband (UWB)-Based Wireless Body Area Networks (WBAN)," in Ultra-Wideband and $60 \mathrm{GHz}$ Communications for Biomedical Applications", Springer US, 2014, pp. 131-152.

[32] A. Segun, A.O. Akinwunmi and E.O. Ogunti, "A Survey of Medium Access Control Protocols in Wireless Sensor Network," Int. Journal of Computer Applications, vol. 116, no. 12, pp. 1-8, April 2015.

[33] H. Cao, V. Leung, C. Chow and H. Chan, "Enabling Technologies for Wireless Body Area Networks: A Survey and Outlook," IEEE Comm. Magazine, pp. 84-93, Dec. 2009.

[34] A. Darif, R. Saadane and D. Aboutajdine, "An Efficient Short Range Wireless Communication Technology for Wireless Sensor Network," in 3rd IEEE International Colloquium on Information Science and Technology, (CIST 2014), Tetouan, Morocco, 20-22 Oct. 2014, pp. 396401.

[35] R. Hunt, "Emerging Wireless Personal Area Networks (WPANs): An analysis of techniques, tools and threats," in $18^{\text {th }}$ IEEE International Conference on Networks (ICON 2012), Singapore, 12-14 Dec. 2012, pp. 274-279.

[36] M. Hämäläinen and J. Iinatti, "Wireless UWB Body Area Networks: Using the IEEE802.15.4-2011," in Series of Academic Press Library in Biomedical Applications of Mobile and Wireless Communications, London, UK, Academic Press, 2014, 48 p.

[37] V. Sipal, B. Allen, D. Edwards and B. Honary, "Twenty years of ultrawideband: Opportunitites and challenges," in IET Communications, vol. 6, no. 10, pp. 1147-1162, July, 2012.

[38] Integrated Project - EUWB, "Ultra-Wideband: Past, Present and Future," White Paper, Presented by the EUWB Consortium, Oct., 2011.

[39] L. Lampe and K. Witrisal, "Challenges and recent advances in IR-UWB system design," in IEEE International Symposium on Circuits and Systems (ISCAS 2010), Paris, France, 30 May - 2 June, 2010, pp. 32883291.

[40] C. Duan, P. Orlik, Z. Sahinoglu and A.F. Molisch, "A Non-Coherent 802.15.4a UWB Impulse Radio," in IEEE International Conference on Ultra-Wideband, (ICUWB 2007), Singapore, 24-26 Sept. 2007, pp. 146151.

[41] G. Dolmans, O. Rousseaux, Li Huang, Ting Fu, B. Gyselinkx, S. D'Amico, A. Baschirotto, J. Ryckaert and B. Van Poucke, "UWB Radio Transceivers For Ultra Low Power and Low Data Rate Communications," in IEEE International Conference on UltraWideband, (ICUWB 2007), Singapore, 24-26 Sept. 2007, pp. 152-157.

[42] V. De Heyn, G. Van der Plas, J. Ryckaert and J. Craninckx, "A fast start-up $3 \mathrm{GHz}-10 \mathrm{GHz}$ digitally controlled oscillator for UWB impulse radio in 90nm CMOS," in 33rd European Solid State Circuits Conference, (ESSCIRC 2007), Munich, Germany, 11-13 Sept. 2007, pp. 484-487.

[43] Y. Takei, H. Katsuta, K. Takizawa, K. Hamaguchi and T. Ikegami, "A prototype of ultra wideband-based wireless body area networks," in IEEE International Conference on Ultra-Wideband (ICUWB 2011), Bologna, Italy, 14-16 Sept. 2011, pp. 420-424.

[44] X. Wang, K. Philips, C. Zhou, B. Busze, H. Pflug, A. Young, J. Romme, P. Harpe, S. Bagga, S. D'Amico, M. De Matteis, A. Baschirotto and H. de Groot, "A high-band IR-UWB chipset for real-time duty-cycled communication and localization systems," in IEEE Asian Solid State Circuits Conference (A-SSCC 2011), Jeju, South-Korea, 14-16 Nov. 2011, pp. 381-384.

[45] J. Rousselot and J.-D. Decotignie, "On the Best Way to Cut a Body Area Network's Wires," in IEEE International Conference on Communications (ICC 2010), Cape Town, South-Africa, 23-27 May 2010, pp.1-5.

[46] M.R. Yuce, K.M. Thotahewa, J-M. Redoute and H. C. Keong, "Development of low-power UWB body sensors," in International Symposium on Communications and Information Technologies (ISCIT 2012), Gold Coast, Australia, 2-5 Oct. 2012, pp.143-148.

[47] X. Lu, X. Chen, Y. Li, D. Jin, L. Zeng and H.F. Rashvand, "ZebraBAN: a heterogeneous high-performance energy efficient wireless body sensor network," in IET Wireless Sensor Systems, vol. 3, no. 4, pp. 247-254, Dec. 2013.

[48] L. Ouvry, G. Masson, M. Pezzin, B. Piaget, B. Caillat, S. Bourdel, N. Dehaese, O. Fourquin, J. Gaubert, S. Meillere and R. Vauche, "A 4GHz CMOS $130 \mathrm{~nm}$ IR-UWB dual front-end transceiver for IEEE802.15 standards," in 21st IEEE International Conference on Electronics, Circuits and Systems (ICECS 2014), Marseille, France, 7-10 Dec. 2014, pp. 798-801.

[49] H. Chougrani, J. Schwoerer, P-H. Horren, A. Baghdadi and F. Dehmas, "UWB-IR digital baseband architecture for IEEE 802.15.6 wireless BAN," in 21st IEEE International Conference on Electronics, Circuits and Systems (ICECS 2014), Marseille, France, 7-10 Dec. 2014, pp. 866869.

[50] D. Morche, G. Masson, S. de Rivaz, F. Dehmas, S. Paquelet, A. Bisiaux, O. Fourquin, J. Gaubert and S. Bourdel, "Double-Quadrature UWB Receiver for Wide-Range Localization Applications With Sub-cm Ranging Precision," in IEEE Journal of Solid-State Circuits, vol. 48, no. 10, pp. 2351-2362, Oct. 2013.

[51] A.R. Mohammed, K. Al-Khateeb and M.R. Islam, "5.5-10.5 GHz IRUWB pulse generator for narrowband interference mitigation," in International Conference on Computer and Communication Engineering (ICCCE 2012), Kuala Lumpur, Malesia, 3-5 July 2012, pp. 55-58.

[52] B. Jajodia, A. Mahanta and R.A. Shaik, "A Six-Segment SRRC Pulse Generator for IEEE 802.15.6 WBAN Standard," in $9^{\text {th }}$ International Conference on Body Area Networks (BODYNETS 2014), London, UK, 29 Sep. - 1 Oct. 2014, pp. 46-49.

[53] S. Kim, Y. Kim, X. Li and J. Kang, "Orthogonal Pulse Design in Consideration of FCC and IEEE 802.15.4a Constraints," in IEEE Communications Letters, vol. 17, no. 5, pp. 896-899, May 2013.

[54] R. Vauche, E. Muhr, N. Tall, A. Haloua, S. Bourdel, J. Gaubert, N. Dehaese and H. Barthelemy, "Ultra-wideband voltage controlled oscillator with commutable phases for BPSK implementation," in IEEE $13^{\text {th }}$ International New Circuits and Systems Conference (NEWCAS 2015), Grenoble, France, 7-10 June 2015, pp.1-4.

[55] A. Dmitriev, A. Laktushkin, Y. Andereyev, A. Kletsov, L. Kuzmin and V. Sinyakin, "UWB direct chaotic transceiver for wireless sensor networks," in 15th IEEE International Conference on Electronics, Circuits and Systems (ICECS 2008), St. Julien's, Malta, 31 Aug. - 3 Sept. 3 2008, pp. 1205-1208.

[56] D. Kreiser and S. Olonbayar, "Design and ASIC implementation of an IR-UWB-baseband transceiver for IEEE 802.15.4a," in International Conference on Wireless Communications and Signal Processing (WCSP 2011), Nanjing, China, 9-11 Nov. 2011, pp. 1-6.

[57] S. Olonbayar, D. Kreiser and R. Kraemer, "FPGA and ASIC implementation and testing of IR-UWB baseband transceiver for IEEE 802.15.4a," in IEEE International Conference on Ultra-WideBand (ICUWB 2014), Paris, France, 1-3 Sept. 2014, pp.456-461.

[58] H.W. Pflug, J. Romme, K. Philips and H. de Groot, "Method to Estimate Impulse-Radio Ultra-Wideband Peak Power," in IEEE Transactions on Microwave Theory and Techniques, vol. 59, no. 4, pp. 1174-1186, April 2011.

[59] N. Soltani, H. Kassiri, H.M. Jafari, K. Abdelhalim and R. Genov, "0.13 $\mu \mathrm{m}$ CMOS $230 \mathrm{Mbps} 21 \mathrm{pJ} / \mathrm{b}$ UWB-IR transmitter with $21.3 \%$ efficiency," in 41st European Solid-State Circuits Conference (ESSCIRC2015), Graz, Austria, 14-18 Sept. 2015, pp. 352-355.

[60] R. Chavez-Santiago, I. Balasingham, J. Bergsland, W. Zahid, K. Takizawa, R. Miura and H-B. Li, "Experimental implant communication of high data rate video using an ultra wideband radio link," in 35th Annual International Conference of the IEEE Engineering in Medicine and Biology Society (EMBC 2013), Osaka, Japan, 3-7 July 2013, pp. 5175-5178. 
[61] D. Anzai, K. Katsu, R. Chavez-Santiago, Q. Wang, D. Plettemeier, J. Wang and I. Balasingham, "Experimental Evaluation of Implant UWBIR Transmission With Living Animal for Body Area Networks," in IEEE Transactions on Microwave Theory and Techniques, vol. 62, no. 1, pp. 183-192, Jan. 2014.

[62] S. Gezici and H.V. Poor, "Position Estimation via Ultra-Wide-Band Signals," in Proceedings of the IEEE, vol. 97, no. 2, pp. 386-403, Feb. 2009.

[63] A. Hernandez, R. Badorrey, J. Choliz, I. Alastruey and A. Valdovinos, "Accurate indoor wireless location with IR UWB systems a performance evaluation of joint receiver structures and TOA based mechanism," in IEEE Transactions on Consumer Electronics, vol. 54, no. 2, pp. 381389, May 2008.

[64] J. Li, X. Cui and C. Wu, "Study on the Ultra-wideband Wireless Positioning Protocol Based on Pseudo-random Turnaround Delay," in Eighth International Conference on P2P, Parallel, Grid, Cloud and Internet Computing (3PGCIC 2013), Compiegne, France, 28-30 Oct. 2013, pp. 369-372.

[65] Z. Sahinoglu, "Improving Range Accuracy of IEEE 802.15.4a Radios In the Presence of Clock Frequency Offsets," in IEEE Communications Letters, vol. 15, no. 2, pp. 244-246, February 2011.

[66] M. Malajner, P. Planinsic and D. Gleich, "UWB ranging accuracy," in International Conference on Systems, Signals and Image Processing (IWSSIP 2015), 10-12 Sept. 2015, pp. 61-64.

[67] Y. Tingcong, M. Walsh and B. O'Flynn, "IEEE 802.15.4a UWB-IR ranging with bilateral transmitter power control methodology for multipath effects mitigation," in 24th IET Irish Signals and Systems Conference (ISSC 2013), Letterkenny, Ireland, 20-21 June 2013, pp. 1-6.

[68] V. Kristem, A.F. Molisch, S. Niranjayan and S. Sangodoyin, "Coherent UWB Ranging in the Presence of Multiuser Interference," in IEEE Transactions on Wireless Communications, vol. 13, no. 8, pp. 44244439, Aug. 2014.

[69] T.V. Nguyen, Y. Jeong, H. Shin and M.Z. Win, "Machine Learning for Wideband Localization", in IEEE Journal on Selected Areas in Communications, vol. 33, no. 7, pp. 1357-1380, July 2015.

[70] I. Tunaru, B. Denis and B. Uguen, "Location-Based Pseudonyms for Identity Reinforcement in Wireless Ad Hoc Networks," in IEEE 81st Vehicular Technology Conference (VTC Spring 2015), Glasgow, UK, 11-14 May 2015, pp. 1-5.

[71] P. Gandolfo, D. Radovic, M. Savic and D. Simic, "IEEE 802.15.4a UWB-IR radio system for telemedicine," in IEEE International Conference on Ultra-Wideband (ICUWB 2008), Hannover, Germany, 10-12 Sept. 2008, pp. 11-14.

[72] M. Hamalainen, A. Taparugssanagorn, R. Tesi and J. linatti, "Wireless medical communications using UWB," in IEEE International Conference on Ultra-Wideband (ICUWB 2009), Vancouver, BC, Canada, 9-11 Sept. 2009, pp. 485-489.

[73] V. Niemela, M. Hamalainen, J. Iinatti and A. Taparugssanagorn, "P-rake receivers in different measured WBAN hospital channels," in 5th International Symposium on Medical Information \& Communication Technology (ISMICT 2011), Montreux, Switzerland, 27-30 March 2011, pp. $42-46$.

[74] V. Niemela, M. Hamalainen and J. Iinatti, "IEEE 802.15.4a UWB receivers in medical applications," Int. J. of Ultra Wideband Communications and Systems, vol. 2, no. 2, 2011, pp. 73-82.

[75] W. Rhee, N. Xu, B. Zhou and Z. Wang, "Low power, non invasive UWB systems for WBAN and biomedical applications," in International Conference on Information and Communication Technology Convergence (ICTC 2010), Jeju, South-Korea, 17-19 Nov. 2010, pp. 3540.

[76] G. Bakul, D. Singh and D. Kim, "Optimized WSN for ECG monitoring in ubiquitous healthcare system," in 4th International Conference on Interaction Sciences (ICIS 2011), Busan, South-Korea, 16-18 Aug. 2011, pp. 23-26.

[77] H.C. Keong and M.R. Yuce, "Analysis of a multi-access scheme and asynchronous transmit-only UWB for wireless body area networks," in Annual International Conference of the IEEE Engineering in Medicine and Biology Society (EMBC 2009), Minneapolis, MN, USA, 3-6 Sept. 2009, pp. 6906-6909.

[78] R. Chavez-Santiago, A. Khaleghi, I. Balasingham and T.A. Ramstad, "Architecture of an ultra wideband wireless body area network for medical applications," in $2^{\text {nd }}$ International Symposium on Applied Sciences in Biomedical and Communication Technologies (ISABEL 2009), Bratislava, Slovakia, 24-27 Nov. 2009, pp. 1-6.
[79] R. Chavez-Santiago, K.E. Nolan, O. Holland, L. De Nardis, J.M. Ferro, N. Barroca,L.M. Borges, F.J. Velez, V. Goncalves and I. Balasingham, "Cognitive radio for medical body area networks using ultra wideband," in IEEE Wireless Communications, vol. 19, no. 4, pp. 74-81, August 2012.

[80] M. Sarestoniemi, V. Niemela, M. Hamalainen, J. Iinatti, N. Keranen, T. Jamsa, J. Partala, T. Seppanen and J. Reponen, "Receiver performance evaluation on IEEE 802.15.6 based WBAN for monitoring Parkinson's disease," in 8th International Symposium on Medical Information and Communication Technology (ISMICT 2014), Florence, Italy, 2-4 April 2014, pp. 1-5.

[81] V. Niemela, M. Hamalainen and J. Iinatti, "On IEEE 802.15.6 IR-UWB receivers - simulations for DBPSK modulation," in 35th Annual International Conference of the IEEE Engineering in Medicine and Biology Society (EMBC 2013), Osaka, Japan, 3-7 July 2013, pp. 16761679.

[82] T. Erseghe and S. Tomasin, "UWB WPAN receiver optimization in the presence of multiuser interference," in IEEE Transactions on Communications, vol. 57, no. 8, pp. 2369-2379, Aug. 2009.

[83] S. Movassaghi, M. Abolhasan, D. Smith and A. Jamalipour, "AIM: Adaptive Internetwork interference mitigation amongst co-existing wireless body area networks," in IEEE Global Communications Conference (GLOBECOM 2014), Austin, TX, USA, 8-12 Dec. 2014, pp. 2460-2465.

[84] M. Hernandez and R. Miura, "Coexistence of IEEE Std 802.15.6 TM $^{\mathrm{TM}} 2012$ UWB-PHY with other UWB systems," in IEEE International Conference on Ultra-Wideband (ICUWB 2012), Syracuse, NY, USA, 17-20 Sept. 2012, pp. 46-50.

[85] J. Choliz, A. Hernandez-Solana, A, Sierra and P. Cluzeaud, "Coexistence of MB-OFDM UWB with Impulse Radio UWB and other radio systems," in IEEE International Conference on Ultra-Wideband (ICUWB 2011), Bologna, Italy, 14-16 Sept. 2011, pp. 410-414.

[86] I. Dotlic, "Interference performance of IEEE 802.15.6 Impulse-Radio Ultra-Wideband physical layer," in IEEE $22^{\text {nd }}$ International Symposium on Personal Indoor and Mobile Radio Communications (PIMRC 2011), Toronto, ON, Canada, 11-14 Sept. 2011, pp. 2148-2152.

[87] Z. Ahmadian and L. Lampe, "Performance of concatenated coded IRUWB in the presence of multiple-access interference," in $42^{\text {nd }}$ Asilomar Conference on Signals, Systems and Computers (ASILOMAR 2008), Pacific Grove, CA, USA, 26-29 Oct. 2008, pp. 2001-2005.

[88] M. Flury, R. Merz and J.-Y. Le Boudec, "Robust IEEE 802.15.4a energy detection receiver using statistical interference modeling," in $43^{\text {rd }}$ Asilomar Conference on Signals, Systems and Computers (ASILOMAR 2009), Pacific Grove, CA, USA, 1-4 Nov. 2009, pp. 952-956.

[89] K. Nakanishi, V. Niemela, M. Hamalainen, J. Iinatti and S. Hara, "On IEEE 802.15.6 IR-UWB ED receiver performance in the presence of multiuser interference," in 9th International Symposium on Medical Information and Communication Technology (ISMICT 2015), Kamakura, Japan, 24-26 March 2015, pp. 204-208.

[90] P. Pelissou, R. Lachaud, P. Dallemagne, J.-D. Decotignie and J.-F. Dufour, "Simulated IEEE 802.15.4a BER results (multipath and coexistence) in the spacecraft environment," in IEEE International Conference on Wireless for Space and Extreme Environments (WiSEE 2014), Noordwijk, Netherlands, 30-31 Oct. 2014, pp. 1-5.

[91] R. Akbar and E. Radoi, "An overview of synchronization algorithms for IR-UWB systems," in International Conference on Computing, Networking and Communications (ICNC 2012), Maui, HI, USA, 30 Jan. - 2 Feb. 2012, pp. 573-577.

[92] R. Reinhold and R. Kays, "Synchronization Performance of IEEE 802.15.4 IR-UWB Systems in Industrial Environments," in German Microwave Conference (GeMIC 2014), Aachen, Germany, 10-12 March 2014, pp. 1-4.

[93] R. Reinhold, R. Kays and I. Kledewski, "CAMDF based synchronization for IEEE 802.15.4 IR-UWB systems in rich scattering environments," in IEEE International Conference on Ultra-WideBand (ICUWB 2014), Paris, France, 1-3 Sept. 2014, pp. 217-221.

[94] R. Reinhold, "Coverage range analysis of IEEE 802.15.4a IR-UWB for reliable data transmission in wireless sensor networks," in IEEE International Workshop on Measurements and Networking Proceedings (M\&N 2013), Naples, Italy, 7-8 Oct. 2013, pp. 31-35.

[95] H. Chougrani, J. Schwoerer, P.-H. Horrein and A. Baghdadi, "Hardware implementation of a non-coherent IR-UWB receiver synchronization algorithm targeting IEEE 802.15.6 wireless BAN," in IEEE International Conference on Ultra-WideBand (ICUWB 2014), Paris, France, 1-3 Sept. 2014, pp. 444-449. 
[96] Z. Zhang, Z, Pang, J. Chen, Q. Chen, H. Tenhunen, L-R. Zheng and X. Yan, "Two-Layered Wireless Sensor Networks for Warehouses and Supermarkets," in Third International Conference on Mobile Ubiquitous Computing, Systems, Services and Technologies, (UBICOMM '09), Sliema, Malta, 11-16 Oct. 2009, pp. 220-224.

[97] D. Dardari, R. D'Errico, C. Roblin, A. Sibille and M.Z. Win, "Ultrawide Bandwidth RFID: The Next Generation?," in Proceedings of the IEEE, vol. 98 , no. 9, pp. 1570-1582, Sept. 2010.

[98] D. Zito, D. Pepe, M. Mincica, F. Zito, D. De Rossi, A. Lanata, E.P. Scilingo and A. Tognetti, "Wearable system-on-a-chip UWB radar for contact-less cardiopulmonary monitoring: Present status," in 30th Annual International Conference of the IEEE Engineering in Medicine and Biology Society (EMBS 2008), Vancouver, BC, Canada, 20-25 Aug. 2008, pp. 5274-5277.

[99] M.S. Mohammadi, E. Dutkiewicz, Q. Zhang and X. Huang, "Optimal Energy Efficiency Link Adaptation in IEEE 802.15.6 IR-UWB Body Area Networks," in IEEE Communications Letters, vol. 18, no. 12, pp. 2193-2196, Dec. 2014.

[100]H. Karvonen, J. Iinatti and M. Hämäläinen, "A cross-layer energy efficiency optimization model for WBAN using IR-UWB transceivers," in Telecommunication Systems, vol 58, no. 2, pp. 165-177, Feb. 2015.

[101]I. Dotlic and Miura, "Chirp Impulse Radio Ultra-Wideband antenna array with low sample-rate digital beam steering," in IEEE 24th International Symposium on Personal Indoor and Mobile Radio Communications (PIMRC 2013), London, UK, 8-11 Sept. 2013, pp. 851855.

[102]T. Kaiser, F. Zheng and E. Dimitrov, "An Overview of Ultra-Wide-Band Systems With MIMO," in Proceedings of the IEEE, vol. 97, no. 2, pp. 285-312, Feb. 2009.

[103]C. J. Mitchell, G. de Abreu and R. Kohno, "Combined Pulse Shape and Pulse Position Modulation for High Data Rate Transmissions in UltraWideband Communications," in Int. J. of Wireless Information Networks, vol. 10, no. 4, pp. 167-178, Oct. 2003.

[104] J. A. Ney da Silva and M.L.R. de Campos, "Performance comparison of binary and quaternary UWB modulation schemes," in IEEE Global Telecommunications Conference, (GLOBECOM '03.), San Francisco, CA, USA, 1-5 Dec. 2003, pp. 789-793.

[105]S. Majhi, A.S. Madhukumar, A.B. Premkumar and F. Chin, "Modulation Schemes Based on Orthogonal Pulses for Time Hopping Ultra Wideband Radio Systems," in IEEE International Conference on Communications, (ICC '07), Glasgow, Scotland, UK, 24-28 June 2007, pp. 4185-4190.

[106]W. Liu, Z. Zhou, Z. Bai, H. Shen, W. Zhang and K. S. Kwak, "A novel UWB modulation method based on time-frequency energy distribution," in IEEE International Symposium on Communications and Information Technology, (ISCIT 2005), Beijing, China, 12-14 Oct. 2005, pp. 960963.

[107]Y. Dai and X. Dong, "Hybrid PPM-BPSK for Transmitted Reference Pulse Cluster Systems in UWB and 60-GHz Channels," in IEEE Wireless Communications Letters, vol. 3, no. 6, pp. 657-660, Dec. 2014.

[108]S. Ji, S. Lee and J. Kim, "Efficient Hybrid Modulation with PhaseDirected Pulse Position Estimation for UWB-IR Systems," in IEEE Transactions on Communications, vol. 61, no. 3, pp. 1171-1177, March 2013.

[109] V. Niemela, M. Hamalainen and J. Iinatti, "Improved usage of time slots of the IEEE 802.15.4a UWB system model," in $14^{\text {th }}$ International Symposium on Wireless Personal Multimedia Communications (WPMC 2011), Brest, France, 3-7 Oct. 2011, pp. 1-5.

[110]T. Paso, V. Niemela, J. Haapola, M. Hamalainen and J. Iinatti, "Performance Evaluation of IEEE 802.15.4-2011 IR-UWB System with Enchanced Modulation Scheme," in $10^{\text {th }}$ International Conference on Body Area Networks (BODYNETS 2015), Sydney, Australia, 28-30 Sept. 2015, pp. 1-7. 